ROBUST SOLUTION OF THE

LINEAR SERVOMECHANISM PROBLEM

by

P.W. Staats, Jr. and J.B. Pearson

September 1974

Technical Report No. 7401 


\section{ROBUST SOLUTION OF THE \\ LINEAR SERVOMECHANISM PROBLEM}

by

P.W. Staats, Jr.* and J.B. Pearson**

\section{ABSTRACT}

This paper furnishes a complete solution to the problem of designing robust controllers for linear servomechanisms. The results complete the work begun in [1] in that arbitrary exogenous signals are included in the problem formulation. Only constant exogenous signals were considered in [1].

* P.W. Staats, Jr., is with MPR Associates, Inc., 1140 Connecticut Ave., N.W., Washington, D.C. 20036.

** J.B. Pearson is with the Department of Electrical Engineering, Rice University, Houston, Texas 77001 .

This work was supported in part by the Fannie and John Hertz Foundation and in part by the National Science Foundation under Grant No. GK-39893. 
In [1], the Robust State Feedback Problem (RSFP) was formulated and solved for a particular case of the linear servomechanism problem in which the exogenous signals to be tracked and/or to be rejected were arbitrary constants. In this paper, the same problem is reformulated and solved for arbitrary exogenous signals, thus completing the theoretical development of robust solutions to the general linear servomechanism problem. We leave the discussion of computational solutions to future articles.

As in [1] we consider the system

$$
\begin{aligned}
& \dot{x}(t)=A x(t)+B u(t) \\
& y(t)=C x(t) \\
& z(t)=D x(t)
\end{aligned}
$$

where $x(t)$ is the state, $u(t)$ the control, $y(t)$ the measured output and $z(t)$ the regulated output.

For the servomechanism problem, we regard the system as being composed of two parts, the plant and the exogenous signals. This endows $A$ and $B$ with a particular structure described as follows. Let $x=x_{1} \oplus x_{2}$ where $x$ is the state space of $(1.1), x_{1}$ is the state space of the plant and $x_{2}$ is the state space of the exogenous signals. Assume $d\left(x_{1}\right)=n_{1}$ and $d\left(x_{2}\right)=n_{2}$. For any $x=x_{1} \oplus x_{2}$, define the maps

$$
\begin{aligned}
& A x=\left(A_{1} x_{1}+A_{3} x_{2}\right) \oplus A_{2} x_{2} \\
& B u=B_{1} u \\
& D x=D_{1} x_{1}+D_{2} x_{2} \\
& C x=C_{1} x_{1}+C_{2} x_{2} \\
& (A+B F) x=\left[\left(A_{1}+B_{1} F_{1} x_{1}+\left(A_{3}+B_{1} F_{2}\right) x_{2}\right] \oplus A_{2} x_{2}\right.
\end{aligned}
$$


These maps have a natural interpretation in matrix terms as follows

$$
\begin{array}{ll}
A \sim\left[\begin{array}{ll}
A_{1} & A_{3} \\
0 & A_{2}
\end{array}\right] & B \sim\left[\begin{array}{l}
B_{1} \\
0
\end{array}\right] . \\
C \sim\left[\begin{array}{ll}
C_{1} & C_{2}
\end{array}\right] & D \sim\left[\begin{array}{ll}
D_{1} & D_{2}
\end{array}\right]
\end{array}
$$

Define the unobservable space of $(C, A)$ by

$$
n=\bigcap_{i=1}^{n} \operatorname{ker} C A^{i-1} \quad \text { where } n=n_{1}+n_{2}
$$

We assume that

$$
\begin{aligned}
& x^{+}(A) \cap x_{1} \subset\langle A \mid B\rangle \\
& x^{+}(A) \cap \eta=0 \\
& \sigma\left(A_{2}\right) \subset C^{+}
\end{aligned}
$$

i.e., our plant is stabilizable, the system is detectable and the exogenous signals are unstable. The problem is not realistic unless (1.4) is true. Detectability is assumed for clarity and by Theorem 4 of [2] represents no loss of generality. No control is required in the servomechanism problem for system modes corresponding to stable eigenvalues of $A_{2}$, so $(1.6)$ is also a reasonable assumption. In addition, we assume that $B_{1}$ is monic and $D_{1}$ is epic.

The Regulator Problem with Internal. Stability (RPIS) was formulated and solved in [2] and is concerned with finding an $F: X \rightarrow U$ such that

$$
\begin{aligned}
& \text { Ker } F \supset \eta \\
& x^{+}(A+B F) \cap(\langle A \mid B\rangle+\eta) \subset \eta \\
& x^{+}(A+B F) \subset \operatorname{ker} D
\end{aligned}
$$


The Robust State Feedback Problem (RSFP) is a generalization of RPIS in that a solution $F: \mathcal{X} \rightarrow \mathcal{U}$ is sought that remains a solution under perturbations. In $\$ 2$, we formulate and solve RSFP; in $\S 3$ we consider state space extension to enlarge the class of systems for which RSFP is solvable and in 84 we consider perturbations in $A$ and $B$. Alternate approaches to this problem are Davison [3] and Francis, Sebakhy and Wonham [4]. The reader is assumed to be familiar with the notation and terminology of [1] and [2].

\section{ROBUST STATE FEEDBACK PROBLEM}

In this section, we consider perturbations in the feedback map $F$ of the form

$$
\tilde{F}=F+\varepsilon \hat{F} /\|F\|
$$

where $\epsilon$ is a scalar and $\hat{F} \neq 0$ is arbitrary. We define admissible perturbations in $F$ as follows. Given $\epsilon_{0}>0$ and $F$ such that $\operatorname{ker} F \supset \eta$. A

perturbation $\tilde{F}$ is admissible if $\operatorname{ker} \hat{F} \supset \eta$ and $0 \leq \varepsilon<\epsilon_{0}$. We now state the main problem under consideration.

\section{ROBUST STATE FEEDBACK PROBLEM (RSFP)}

Given the maps $\mathrm{A}: \mathcal{X} \rightarrow x, \mathrm{~B}: \mathcal{U} \rightarrow X, \mathrm{D}: \mathcal{X} \rightarrow Z$, and a subspace $\eta \subset x$ such that $A \eta \subset \eta$. Under what conditions do there exist $F: x \rightarrow u$ and $\varepsilon_{0}>0$ such that with Ker $F \supset \eta$

$$
\begin{aligned}
& x^{+}(A+B \tilde{F}) \subset \operatorname{Ker} D \\
& x^{+}(A+B \tilde{F}) \cap\langle A \mid B\rangle=0
\end{aligned}
$$

for every admissible $\tilde{F}$.

RPIS is clearly a special case of RSFP when $\epsilon=0 .(2.3)$ is simpler than (1.8) because of our special assumption (1.5). The solution of RSFP is 
given by

Theorem 2.1

Assume (1.4) - (1.6) are true. RSFP is solvable if and only if

(i) RPIS is solvable

and

(ii) for any solution $F: x \rightarrow U$ to RPIS

$B \subset \bigcap_{j=1}^{k}\left(A+B F-\lambda_{i}\right)^{j} \operatorname{ker} D$

for each $\lambda_{i} \in \sigma\left(A_{2}\right)$, where $k_{i}$ is the multiplicity of $\lambda_{i}$ as a root of the minimal polynomial of $A_{2}$.

Remark: In $[1], A_{2}=0, k_{i}=1$ so (2.4) reduced to $B \subset(A+B F) \operatorname{Ker} D$.

The proof of Theorem 2.1 is given in the Appendix.

This Theorem is not convenient to apply in its present form, but with an additional assumption can be shown to be equivalent to a set of matrix rank conditions. These conditions are easier to apply to problem solving and lead also to a natural extension algorithm to be discussed in $\S 3$. Our additional assumption involves the structure of the matrices $A_{3}$ and $D_{2}$ which describe how the exogenous signals are coupled into the plant and the regulated output. Assume that the matrix of $A_{2}$ has been placed in Jordan normal form and the Jordan blocks have been ordered in the following way. Assume $\sigma\left(A_{2}\right)=$ $\left\{\lambda_{1}, \lambda_{2}, \ldots, \lambda_{r}\right\}$ and define

$$
q_{i} \equiv d\left(\operatorname{ker}\left(A_{2}-\lambda_{i}\right)\right)
$$


Then $q_{i}$ is the number of cyclic subspaces of $x_{2}$ corresponding to $\lambda_{i}$. These subspaces can be written as $x_{2 i}^{l}, \ldots, x_{2 i}^{q}$. where we define

$$
k_{i j} \equiv d\left(X_{2 i}^{j}\right)
$$

Furthermore, these subspaces will be indexed so that $k_{i l} \geq k_{i 2} \geq \ldots k_{i q}$. Corresponding to this ordering, the matrices of $A_{3}$ and $D_{2}$ are partitioned as follows

$$
\begin{aligned}
& A_{3}=\left[\begin{array}{llll}
A_{11} & A_{12} & \cdots & A_{r q_{r}}
\end{array}\right] \\
& D_{2}=\left[\begin{array}{llll}
D_{11} & D_{12} & \cdots & D_{r q_{r}}
\end{array}\right]
\end{aligned}
$$

where the partitioned matrices have the structure

$$
\begin{aligned}
& A_{i j}=\left[\begin{array}{llll}
a_{i j} & 0 & \ldots & 0
\end{array}\right] \\
& D_{i j}=\left[\begin{array}{llll}
d_{i j} & 0 & \ldots & 0
\end{array}\right]
\end{aligned}
$$

Then using the matrix representations indicated by (1.3), the solvability conditions for RSFP can be stated in the following way.

\section{Corollary 2.1}

Assume (1.4) - (1.6) and (2.7) are true, RSFP is solvable if and only if

$$
\begin{aligned}
& \operatorname{rank}\left[\begin{array}{cc}
\left(A_{1}-\lambda_{i} 1\right) & B_{1} \\
D_{1} & 0
\end{array}\right]=\operatorname{rank}\left[\begin{array}{ccc}
\left(A_{1}-\lambda_{i} I\right) & B_{1} & a_{i j} \\
D_{1} & 0 & d_{i j}
\end{array}\right]=n_{1}
\end{aligned}
$$

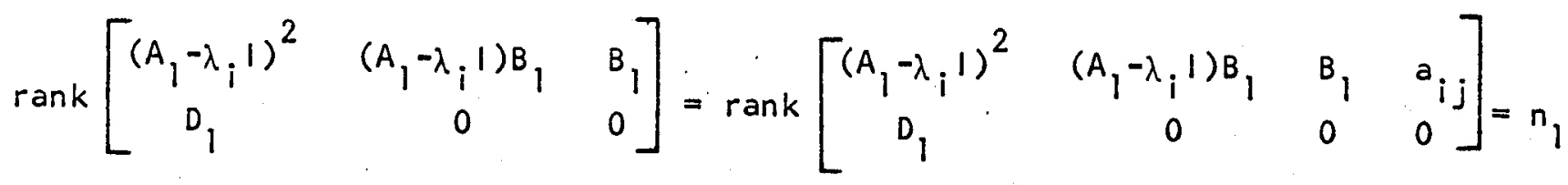




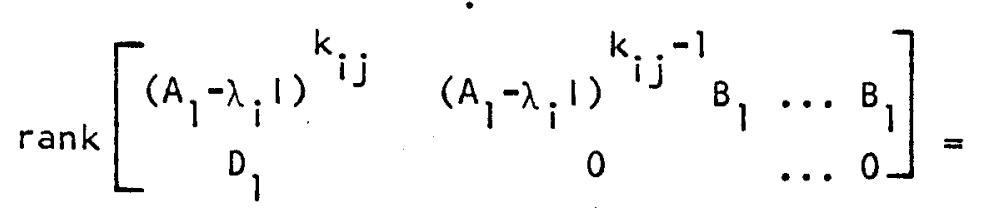

$$
\begin{aligned}
& \operatorname{rank}\left[\begin{array}{cccc}
\left(A_{1}-\lambda_{i} 1\right)^{k_{i j}} & \left(A_{1}-\lambda_{i} 1\right)^{k_{i j}{ }^{-I_{B}}} & \ldots B_{1} & a_{i j} \\
D_{1} & 0 & 0 & 0
\end{array}\right]=n_{1}
\end{aligned}
$$

for $\quad i \in \bar{r} \equiv(1,2, \ldots, r)$

$$
j \in \bar{q}_{i} \equiv\left(1,2, \ldots, q_{i}\right)
$$

The proof is given in the Appendix and yields an interesting result which we state as

\section{Corollary 2.2}

Assume (1.4) - (1.6) are true. If RSFP has a solution, then any admissible $F: X \rightarrow U$ satisfying $X^{+}(A+B F) \cap\langle A \mid B\rangle=0$ is a solution.

This simply means that if RSFP has a solution, then any admissible. F that stabilizes the plant is a solution.

\section{STATE SPACE EXTENSION}

There will be systems for which RSFP fails to have a solution and Corollary 2.1 shows that this can happen because of the failure of either or both equalities of (2.8). From the proof of this Corollary it follows that the first equality is a consequence only of solvability of RPIS. Thus, if this equality fails there is no solution. This follows because if RPIS is not solvable, then ERPIS (Extended RPIS) is not solvable [2]. However, there will be cases for which problems only occur in the second equality of some of the equations of 
(2.8). In these cases there is hope for solution via state space extension.

The state space extension to be developed is defined as follows: Let $x^{e}=x \oplus x_{3}$ and the extended maps have the properties

$$
A^{e}: X^{e} \rightarrow x^{e}, A^{e}\left(x \oplus x_{3}\right)=A x \oplus L\left(x \oplus x_{3}\right)
$$

where $\quad L: x^{e} \rightarrow x_{3}$

$$
\begin{aligned}
& B^{e}: U \rightarrow x^{e}, B^{e} u=B u \\
& C^{e}: x^{e} \rightarrow y^{e}, C^{e}\left(x \oplus x_{3}\right)=C x \oplus x_{3} \\
& D^{e}: x^{e} \rightarrow Z, D^{e}\left(x \oplus x_{3}\right)=D x
\end{aligned}
$$

where $\quad x \in X, x_{3} \in x_{3}, u \in U$. The state space of the "extended plant" is $x_{1}^{\mathrm{e}}=x_{1} \oplus x_{3}$ and the maps have matrix representations as follows

$$
\begin{array}{ll}
A_{1}^{e}=\left[\begin{array}{ll}
A_{1} & 0 \\
L_{1} & L_{2}
\end{array}\right] & A_{3}^{e}=\left[\begin{array}{l}
A_{3} \\
L_{3}
\end{array}\right] \\
D_{1}^{e}=\left[\begin{array}{ll}
D_{1} & 0
\end{array}\right] & D_{2}^{e}=\left[D_{2}\right] \\
C_{1}^{e}=\left[\begin{array}{ll}
C_{1} & 0 \\
0 & 1
\end{array}\right] & L=\left[\begin{array}{lll}
0 & 0 & 0 \\
L_{1} & L_{2} & L_{3} \\
0 & 0 & 0
\end{array}\right]
\end{array}
$$

where $L_{1}, L_{2}$, and $L_{3}$ must be determined. Thus, the maps of the extended system are specified by (3.1) and the Extended Robust State Feedback Problem is defined as follows:

\section{EXTENDED ROBUST STATE FEEDBACK PROBLEM (ERSFP)}

Given the maps $\mathrm{A}: x \rightarrow x, \mathrm{~B}: \mathcal{U} \rightarrow x, \mathrm{c}: x \rightarrow y$, and $D: x \rightarrow z$, under what conditions does there exist a state space extension (i.e., a map $L: x^{e} \rightarrow x_{3}$ ) such that RSFP is solvable for $\left(A^{e}, B^{e}, C^{e}, D^{e}\right)$. 
It is clear that a necessary condition for a solution to ERSFP to exist is that RPIS be solvable. It will be shown that this is also a sufficient condition and the extension that solves ERSFP is developed via the presentation of an algorithm, the Extension Algorithm, that systematically investigates and corrects the rank conditions of $(2.8)$.

Three lemmas are used in developing the Extension Algorithm.

\section{Lemma 3.1}

Assume that $\left(A_{1}, B_{1}\right)$ is stabilizable. For any $\lambda \in C^{+}$and any positive. integer $k$

$$
\operatorname{rank}\left[\left(A_{1}-\lambda 1\right)^{k} \quad\left(A_{1}-\lambda 1\right)^{k-1} B_{1} \cdots\left(A_{1}-\lambda 1\right) B_{1} B_{1}\right]=n_{1}
$$

Lemma 3.2

Assume that $\left(A_{1}, B_{1}\right)$ is stabilizable and $\lambda \in \mathbb{C}^{+}$. Let $\bar{D}_{1}$ be an $s \times n_{1}$ matrix of rank $s$ and $j$ the smallest integer such that

$$
\operatorname{rank}\left[\begin{array}{ccccc}
\left(A_{1}-\lambda l\right)^{j} & \left(A_{1}-\lambda 1\right)^{j-B_{B}} & \ldots & \left(A_{1}-\lambda 1\right) B_{1} & B_{1} \\
D_{1} & 0 & \ldots & 0 & 0
\end{array}\right]=n_{1}+s
$$

then

$$
\operatorname{rank}\left[\begin{array}{ccccc}
\left(A_{1}-\lambda I\right)^{t} & \left(A_{1}-\lambda I\right)^{t-1} B_{1} & \ldots & \left(A_{1}-\lambda I\right) B_{1} & B_{1} \\
D_{1} & 0 & \ldots & 0 & 0
\end{array}\right]=n_{1}+s
$$

for all $t>j$

The proofs of these lemmas are straightforward and are left to the reader. Lemma 3.3

Given $(A, B, C)$ where $A$ is an $n \times n$ matrix 


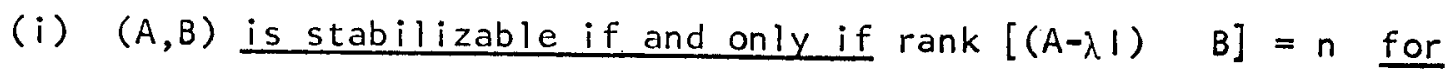
all $\lambda \in \mathrm{C}^{+}$.

(ii) $(A, C)$ is detectable if and only if $\operatorname{rank}\left[\begin{array}{c}(A-\lambda \mid) \\ C\end{array}\right]=n$ for all $\lambda \in \mathrm{c}^{+}$.

The proof of ( $i$ ) is straightforward and that of ( $i i)$ follows by duality.

\section{THE EXTENSION AL GOR ITHM}

\section{Step 1}

This step examines the first rank condition of (2.8) for each $\lambda_{i} \in \sigma\left(A_{2}\right)$ and the plant is extended as required. The procedure begins by looking at the first rank condition and $\lambda_{1}$, i.e., consider

$$
\operatorname{rank}\left[\begin{array}{cc}
\left(A_{1}-\lambda_{1} 1\right) & B_{1} \\
D_{1} & 0
\end{array}\right]=n_{1}+\bar{t}
$$

By Lemma $3.1, \bar{t} \geq 0$ and if $\bar{t}=0$ no extension is required. If an extension is not necessary, the algorithm next considers, $\lambda_{2}$ and the first rank condition. Suppose $\lambda_{i}$ is the first element of $\sigma\left(A_{2}\right)$ requiring extension in this step. Then

$$
\operatorname{rank}\left[\begin{array}{cc}
\left(A_{1}-\lambda_{i} 1\right) & B_{1} \\
D_{1} & 0
\end{array}\right]=n_{1}+\bar{t} \quad \bar{t}>0
$$

and by Lemma 3.1 there are $\bar{t}$ outputs represented by $\bar{D}=\left[\begin{array}{lll}\bar{D}_{1} & \bar{D}_{2}\end{array}\right]$ such that

$$
\operatorname{rank}\left[\begin{array}{cc}
\left(A_{1}-\lambda_{i} I\right) & B_{1} \\
D_{1} & 0
\end{array}\right]=n_{1}+\bar{t}
$$

Note that by Lemma 3.2 all $k_{i}=k_{i l}$ rank conditions fail for these $\bar{t}$ outputs, i.e., 


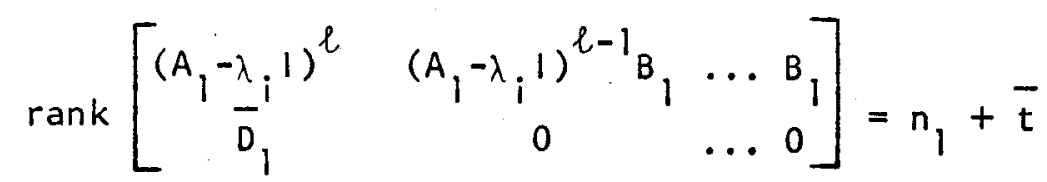

for $1 \leq l \leq k_{i}$. The system is extended by $\bar{t} k_{i}$ dimensions to give $n_{1}^{e}=$ $n_{i}+\bar{t} k_{i}$ and the extended maps have the representations

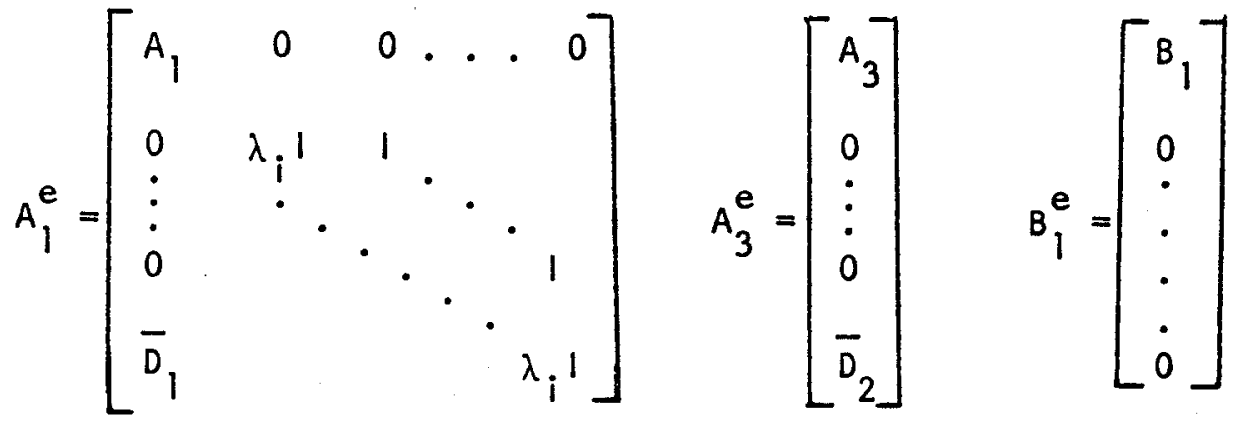

$$
\begin{aligned}
& D_{1}^{e}=\left[\begin{array}{llll}
D_{1} & 0 & \ldots & .
\end{array}\right] \quad D_{2}^{e}=\left[D_{2}\right] \\
& c_{1}^{e}=\left[\begin{array}{ccccccc}
c_{1} & 0 & \cdots & . & . & 0 \\
0 & 1 & & & & \\
\cdot & & & & & & \\
\cdot & & & \cdot & & \\
\cdot & & & & \cdot & \\
0 & & & & & 1
\end{array}\right] \quad c_{2}^{e}=\left[\begin{array}{c}
c_{2} \\
0 \\
\cdot \\
\cdot \\
0
\end{array}\right]
\end{aligned}
$$

The detectability of the extended system and the stabilizability of the extended plant follow from Lemma 3.3. Thus the assumptions (1.4) - (1.6) remain valid for the extended system.

From (3.3) - (3.5) it is clear that this extension corrects the rank condition of concern, i.e., now

$$
\operatorname{rank}\left[\begin{array}{cc}
\left(A_{i}^{e}-\lambda_{i} 1\right) & B_{i}^{e} \\
D_{l}^{e} & 0
\end{array}\right]=\operatorname{rank}\left[\begin{array}{ccc}
\left(A_{i}^{e}-\lambda_{i} l\right) & B_{i}^{e} & a_{i j}^{e} \\
D_{i}^{e} & 0 & d_{i j}^{e}
\end{array}\right]=n_{l}^{e}
$$

for each $j \in \bar{q}_{i}$. Simple matrix manipulations also show that the outputs involved in the extension of (3.5), i.e., those represented by $\bar{D}$, now satisfy 
all rank conditions of $(2.8)$ with respect to $\lambda_{i}$. Thus these outputs need not be checked for $\lambda_{i}$ in any of the subsequent steps of the algorithm.

The maps $A_{1}, A_{3}, B_{1}$, etc. are now redefined as $A_{1} \equiv A_{1}^{e}, A_{3} \equiv A_{3}^{e}$, $B_{1} \equiv B_{1}^{e}$, etc. and $n_{1}$ is redefined as $n_{1} \equiv n_{1}^{e}$. Since RPIS remains solvable and the assumptions $(1.4)-(1.6)$ are still valid, the situation is equivalent to the one existing prior to extension except that an additional rank condition is now satisfied. Next the first rank condition is examined for $\lambda_{i+1}$ and if extension is necessary, it is accomplished as in (3.5) using $\lambda_{i+1}$ instead of $\lambda_{i}$ and the appropriate outputs. This systematic procedure is continued through $\lambda_{r}$ and clearly the first rank condition is now equal to $n_{1}$ for all $\lambda_{i} \in \sigma\left(A_{2}\right)$ where $n_{1}$ may have been redefined several times. This completes step 1.

\section{$\underline{\text { Step } 2}$}

This step examines the second rank condition of (2.8) for each $\lambda_{i} \in \sigma\left(A_{2}\right)$ beginning with $\lambda_{1}$. Suppose that $\lambda_{i}$ for some $i \in \bar{r}$ is the first element of $\sigma\left(A_{2}\right)$ requiring extension, then

$$
\operatorname{rank}\left[\begin{array}{ccc}
\left(A_{1}-\lambda_{i} 1\right)^{2} & \left(A_{1}-\lambda_{i} 1\right) B_{1} & B_{1} \\
D_{1} & 0 & 0
\end{array}\right]=n_{1}+\hat{t}
$$

and there are $\hat{\mathrm{t}}$ outputs represented by $\hat{D}=\left[\begin{array}{ll}\hat{D}_{1} & \hat{D}_{2}\end{array}\right]$ such that

$$
\operatorname{rank}\left[\begin{array}{ccc}
\left(A_{1}-\lambda_{i} 1\right)^{2} & \left(A_{1}-\lambda_{i} 1\right) B_{1} & B_{1} \\
D_{1} & 0 & 0
\end{array}\right]=n_{1}+\hat{t}
$$

From step $1 \operatorname{rank}\left[\begin{array}{cc}\left(A_{1}-\lambda_{i}()\right. & B_{1} \\ \hat{D}_{1} & 0\end{array}\right]=n_{1}$ and since $\left(A_{1}, B_{1}\right)$ is stabilizable, there exists a $\hat{\mathrm{t}} \times \mathrm{n}_{1}$ matrix $G$ such that

$$
G\left(A_{1}-\lambda_{i} I\right)=\hat{D}_{1} \quad G B=0
$$


Furthermore, $G$ has the property that

$$
\operatorname{rank}\left[\begin{array}{cc}
\left(A_{1}-\lambda_{i} 1\right) & B_{1} \\
G & 0
\end{array}\right]=n_{1}+\hat{t}
$$

as can be seen from noting that

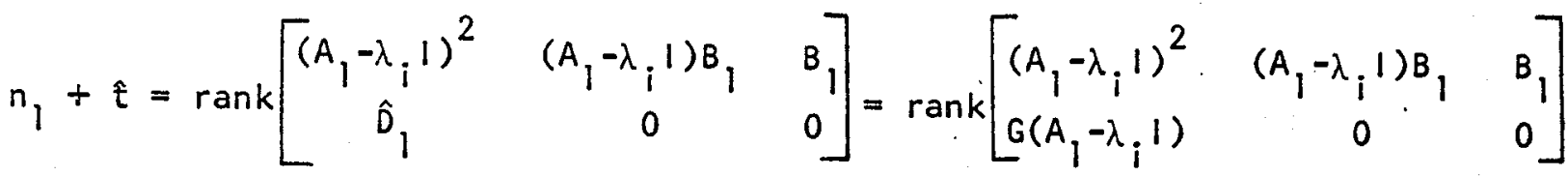

$$
\begin{aligned}
& =\operatorname{rank}\left[\begin{array}{cc}
\left(A_{1}-\lambda_{i} 1\right) & B_{1} \\
G & 0
\end{array}\right]\left[\begin{array}{ccc}
\left(A_{1}-\lambda_{i} 1\right) & B_{1} & 0 \\
0 & 0 & 1
\end{array}\right]
\end{aligned}
$$

and recalling that the rank of the product of two matrices can be no greater than that of either of its factors.

Lemma 3.2 implies that the remaining $k_{i}^{-l}$ rank conditions fail for the $\hat{\mathrm{t}}$ outputs represented by $\hat{b}$. Thus, an extension of dimension $\hat{\mathbf{t}}\left(\mathrm{k}_{\mathbf{i}}-1\right)$ is required and the system is extended to give $n_{1}^{e}=n_{1}+\hat{t}\left(k_{i}-1\right)$ and

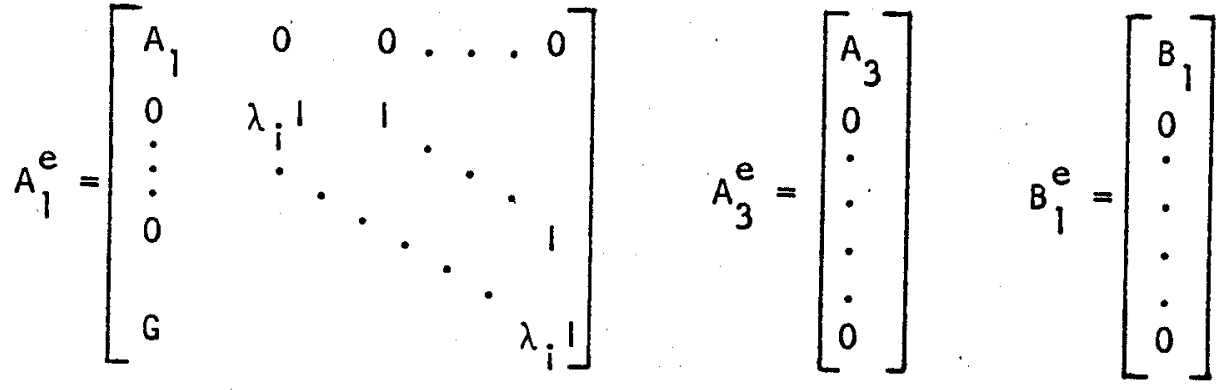

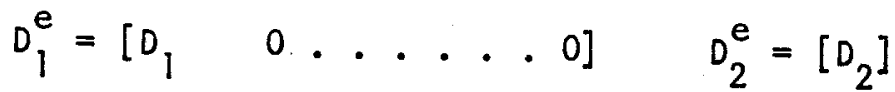

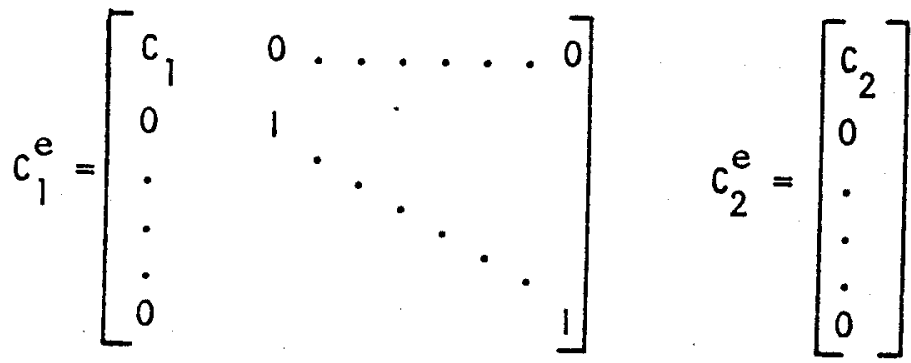

The detectability of the extended system follows directly from Lemma 3.3, and equation (3.9) and Lemma 3.3 combine to yield plant stabilizability. Thus the 
assumptions of (1.4) - (1.6) remain valid.

To see that the extension corrected the rank condition of concern, observe that

$$
\begin{aligned}
& \operatorname{rank}\left[\begin{array}{cccc}
\left(A_{1}^{e}-\lambda_{i} 1\right)^{2} & \left(A_{i}^{e}-\lambda_{i} 1\right) B_{i}^{e} & B_{1}^{e} & a_{i j}^{e} \\
D_{1}^{e} & 0 & 0 & 0
\end{array}\right]=
\end{aligned}
$$

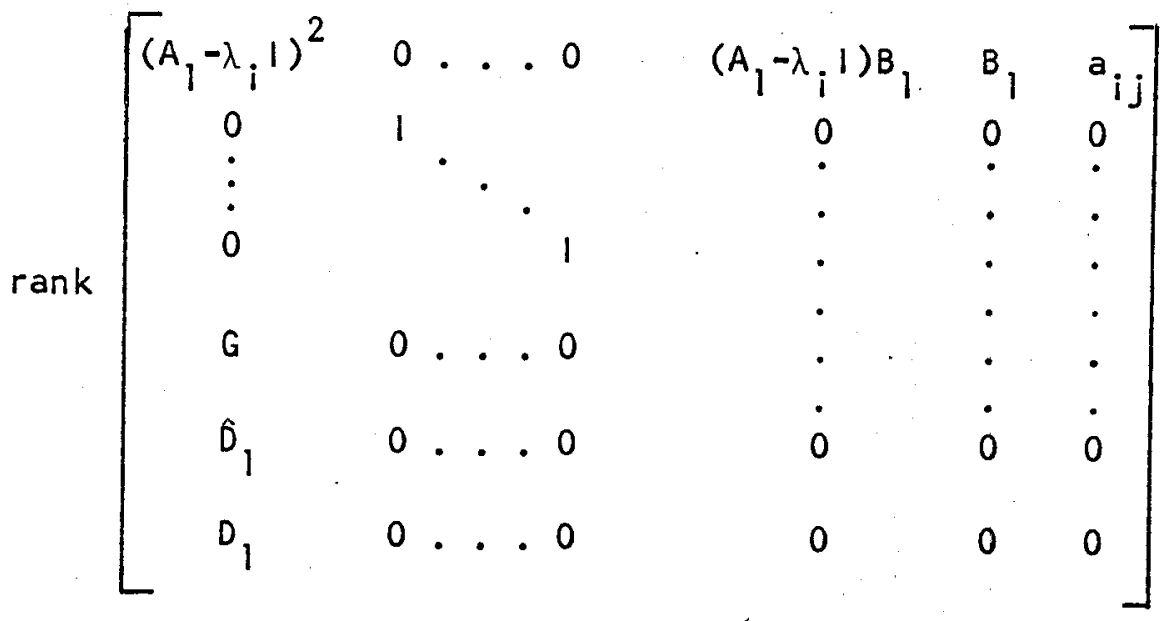

$$
\begin{aligned}
& =\operatorname{rank}\left[\left(A_{1}^{e}-\lambda_{i} 1\right)^{2} \quad\left(A_{1}^{e}-\lambda_{i} 1\right) B_{1}^{e} \quad B_{1}^{e} \quad a_{i j}^{e}\right]
\end{aligned}
$$

and since $\left(A_{1}^{e}, B_{1}^{e}\right)$ is stabilizable, Lemma 3.1 implies

$$
\begin{aligned}
\operatorname{rank}\left[\begin{array}{ccc}
{\left[A_{1}^{e}-\lambda_{i}\right)^{2}} & \left(A_{1}^{e}-\lambda_{i} 1\right) B_{1}^{e} & B_{1}^{e} \\
D_{1}^{e} & 0 & 0
\end{array}\right] & =\operatorname{rank}\left[\begin{array}{cccc}
\left(A_{1}^{e}-\lambda_{i} 1\right)^{2} & \left(A_{1}^{e}-\lambda_{i} 1\right) B_{1}^{e} & B_{1}^{e} & a_{i j}^{e} \\
D_{1}^{e} & 0 & 0 & 0
\end{array}\right] \\
& =n_{1}^{e}
\end{aligned}
$$

for each $j \in \bar{q}_{i}$. Similar matrix manipulations also show that the outputs involved in the extension of (3.10), i.e., those represented by $\hat{D}$, now satisfy all rank conditions of $(2.8)$ with respect to $\lambda_{i}$. Thus these outputs need not be checked for $\lambda_{i}$ in any of the subsequent steps of the algorithm.

The maps $A_{1}, A_{3}, B_{1}$, etc. are now redefined as $A_{1} \equiv A_{1}^{e}, A_{3} \equiv A_{3}^{e}, B_{1} \equiv B_{1}^{e}$, etc. and $n_{1}$ is redefined as $n_{1} \equiv n_{1}^{e}$. Since RPIS remains solvable and the assumptions of $(1.4)-(1.6)$ are still valid, the situation is equivalent to 
the one existing prior to extension except that an additional rank condition is satisfied. Next, the second rank condition is examined for $\lambda_{i+1}$ and if extension is necessary it is accomplished using the technique of (3.7) - (3.10). This procedure is continued in the same systematic manner as in step 1 and it is clear that at the end of step 2 the second rank condition of (2.8) is equal to $n_{1}$ for all $\lambda_{i} \in \sigma\left(A_{2}\right)$ where $n_{1}$ may have been redefined several times.

\section{Step 3}

This step examines the third rank condition of (2.8) in the same manner as the two previous steps. The only difference between the technique of step 2 and that of step 3 is the following: Suppose the $t^{\prime \prime}$ outputs represented by $D^{\prime \prime}=\left[\begin{array}{ll}D_{1}^{\prime \prime} & D_{2}^{\prime \prime}\end{array}\right]$ are such that

$$
\begin{aligned}
& \operatorname{rank}\left[\begin{array}{cccc}
\left(A_{1}-\lambda_{i} I\right)^{3} & \left(A_{1}-\lambda_{i} 1\right)^{2} B_{1} & \left(A_{1}-\lambda_{i} I\right) B_{1} & B_{1} \\
D_{1} & 0 & 0 & 0
\end{array}\right]= \\
& \operatorname{rank}\left[\begin{array}{cccc}
\left(A_{1}-\lambda_{i} 1\right)^{3} & \left(A_{1}-\lambda_{i} I\right)^{2} B_{1} & \left(A_{1}-\lambda_{i} I\right) B_{1} & B_{1} \\
D_{1}^{\prime \prime} & 0 & 0 & 0
\end{array}\right]=n_{1}+t^{\prime \prime}
\end{aligned}
$$

Then the extension is of dimension $t^{\prime \prime}\left(k_{i}-2\right)$ and the $t^{\prime \prime} \times n_{1}$ matrix $G^{\prime \prime}$ is determined by

$$
\operatorname{rank}\left[\begin{array}{ccc}
\left(A_{1}-\lambda_{i} 1\right)^{2} & \left(A_{1}-\lambda_{i} \mid\right) B_{1} & B_{1} \\
D_{1}^{\prime \prime} & 0 & 0
\end{array}\right]=n_{1}
$$

i.e.,

$$
G^{\prime \prime}\left(A_{1}-\lambda_{i} 1\right)^{2}=D_{1}^{\prime \prime} \quad G^{\prime \prime}\left(A_{1}-\lambda_{i}{ }^{\prime}\right) B_{1}=G^{\prime \prime} B_{1}=0
$$

The algorithm continues through as many steps as necessary, the maximum being $\max _{i \in \bar{r}} k_{i}$ and upon completion, all rank conditions of (2.8) are equal 
to $n_{1}$ (where the system maps and $n_{1}$ have been redefined several times).

This procedure is a constructive proof of the following.

Theorem 3.1

Assume that $(1.4)-(1.6)$ and (2.7) are true and RSFP is not solvable. ERSFP is solvable if and only if RPIS is solvable.

The Extension Algorithm does not differentiate between real and complex roots of the minimal polynomial of $A_{2}$. However, any extension that is actually realized must use only real elements. This difficulty is resolved by application of a simple coordinate transformation to the extension developed by the Extension Algorithm. This follows because the roots of the minimal polynomial of $A_{2}$ occur in complex conjugate pairs and a simple calculation shows that if a rank condition fails for some group of outputs and $\lambda_{i}$ then it also fails for the same group of outputs and $\lambda_{i}^{*}$. Now suppose that some rank condition failed for a group of outputs represented by $\bar{D}, \lambda_{i}$, and $\lambda_{i}^{*}$. Then the combined extension is

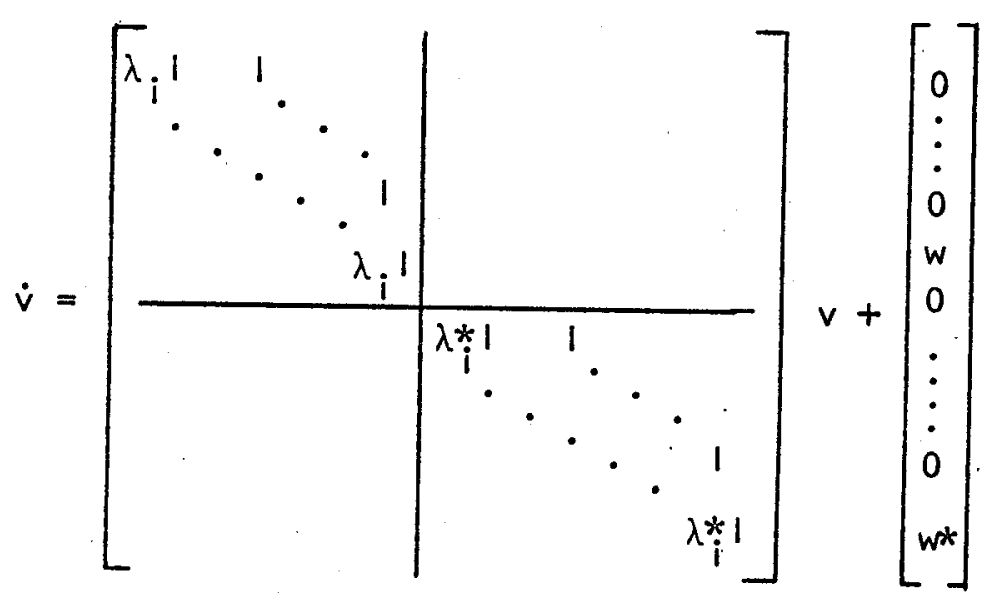

where $w=w^{*}=\overline{D x}$ if step I was used or $w=\left[\begin{array}{lll}G & 0 \ldots\end{array}\right] x$ and the matrix G was determined by an equation like (3.8) or (3.11) if step 1 was not used. 
The state variables of the extension can be redefined using $\hat{T} v=\hat{v}$ where the transformation matrix $\hat{\mathrm{T}}$ is given by

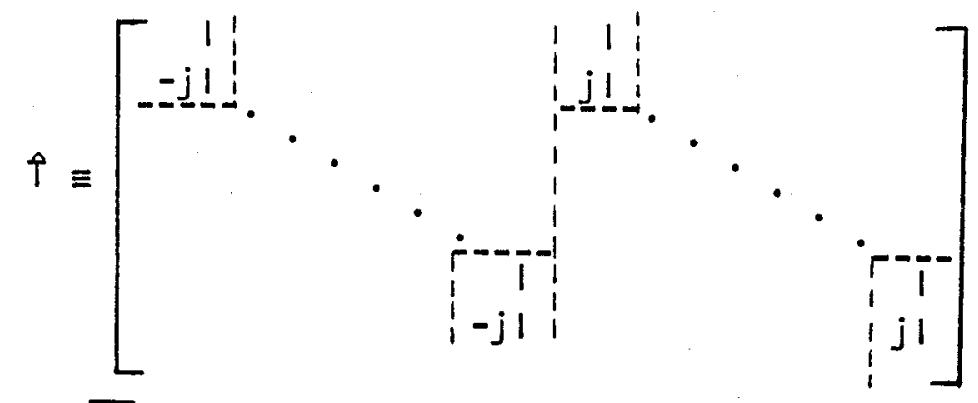

where $j=\sqrt{-1}$. Then the extension is represented by

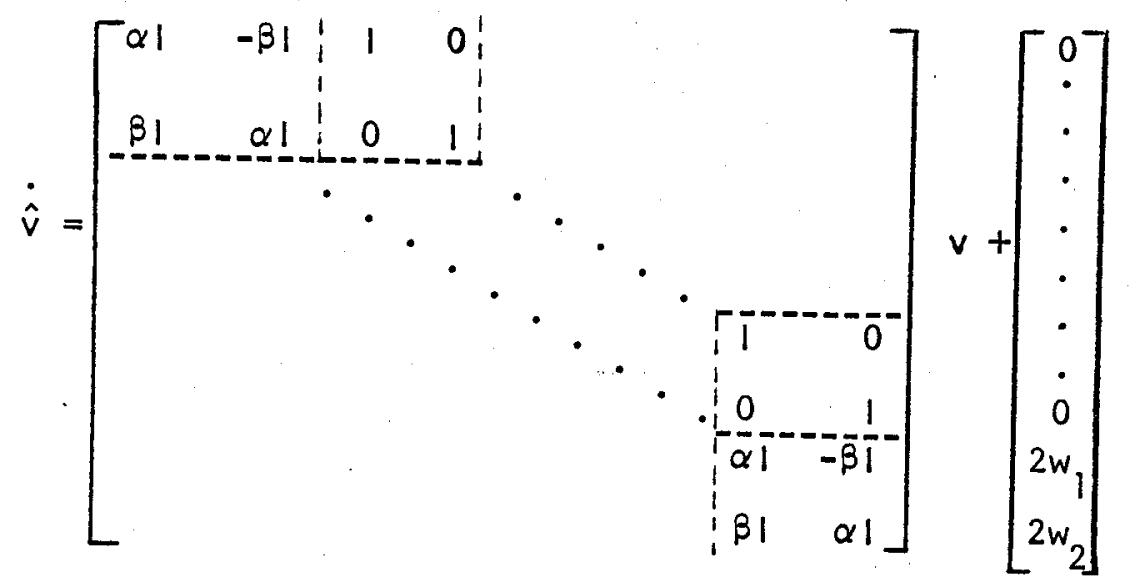

where $\lambda_{i}=\alpha+j \beta, w=w_{1}+j w_{2}$, and the extension is expressed in a form utilizing only real numbers.

It appears that certain measurability assumptions are required to realize the extensions developed by the Extension Algorithm. However, the detectability of the system always permits the extensions to be realized using measurable outputs. For example, suppose that the matrix $G$ defined by (3.8) yields some variables that are not measurable. Since $(A, C)$ is detectable, Lemma 3.3 gives

$$
\operatorname{rank}\left[\begin{array}{cccc}
\left(A_{1}-\lambda_{i} 1\right) & a_{i 1} & \cdots & a_{i q_{i}} \\
c_{1} & c_{i 1} & \cdots & c_{i q_{i}}
\end{array}\right]=n_{1}+q_{i}
$$


where the $c_{i j}$ are defined as in (2.7), i.e., the matrix has full column rank and so

$$
\operatorname{rank}\left[\begin{array}{cccc}
\left(A_{1}-\lambda_{i}{ }^{1}\right) & a_{i 1} & \cdots & a_{i q_{i}} \\
c_{1} & c_{i 1} & \cdots & c_{i q_{i}}
\end{array}\right]=\operatorname{rank}\left[\begin{array}{cccc}
\left(A_{1}-\lambda_{i} 1\right) & a_{i 1} & \ldots & a_{i q_{i}} \\
c_{1} & c_{i 1} & \cdots & c_{i q_{i}} \\
G & 0 & \ldots & 0
\end{array}\right]
$$

Then equation (3.12) implies that there exist matrices $H_{1}$ and $\bar{H}_{1}$ such that

$$
\begin{aligned}
& \bar{H}_{1}\left(A_{1}-\lambda_{i} l\right)+H_{1} C_{1}=G \\
& \bar{H}_{1} a_{i j}+H_{1} c_{i j}=0 \quad j \in \bar{q}_{i}
\end{aligned}
$$

Continuing in this manner it is possible to define matrices $\mathrm{H}_{l}$ and $\vec{H}_{l}$ having the properties

$$
\begin{aligned}
& \bar{H}_{l+1}\left(A_{1}-\lambda_{i} I\right)+H_{l+1} C_{l}=\bar{H}_{l} \\
& \bar{H}_{l+1} a_{i j}+H_{l+1} c_{i j}=0 \quad j \in \bar{q}_{i}
\end{aligned}
$$

for $\ell \in \overline{\mathrm{k}_{\mathrm{i}}-2}$. Observe that the $H_{\ell}$ determined in this manner can be used to define measurable variables

$$
w_{l} \equiv H_{l} C x \quad l \in \overline{k_{i}-1}
$$

and the extension of $(3.10)$ is realized as

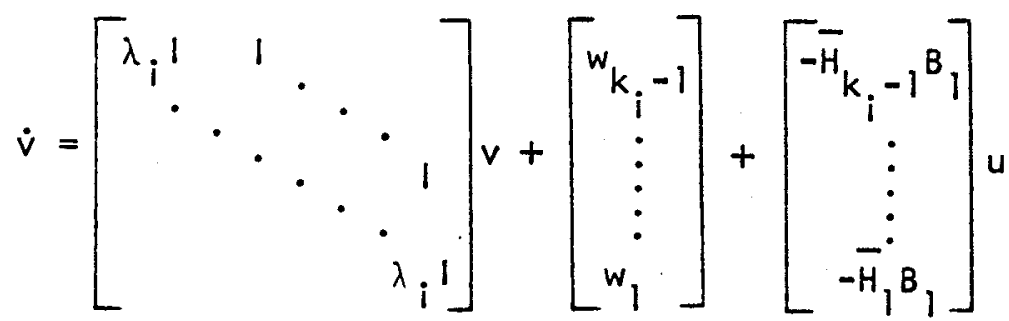

- Note that only measurable variables are used to couple this form of the extension to the system and the extended system is 
18

$$
\begin{aligned}
& A_{1}^{e}=\left[\begin{array}{ccccc}
A_{1} & 0 & 0 & \cdots & 0 \\
H_{k_{i}-1} C_{1} & \lambda_{i}{ }^{\prime} & 1 & & \\
\vdots & & & \cdot & 1 \\
H_{1} C_{1} & & & & \lambda_{i}{ }^{1}
\end{array}\right] \\
& a_{i j}^{e}=\left[\begin{array}{c}
a_{i j} \\
H_{k_{i}}-1 c_{i j} \\
\vdots \\
H_{1} c_{i j}
\end{array}\right] \\
& B_{1}^{e}=\left[\begin{array}{c}
B_{1} \\
-\bar{H}_{k_{i}-1} B_{1} \\
\vdots \\
-\bar{H}_{1} B_{1}
\end{array}\right] \\
& D_{1}^{e}=\left[D_{1}\right. \\
& \text { o..... 0] } \\
& d_{i j}^{e}=\left[d_{i j}\right]
\end{aligned}
$$

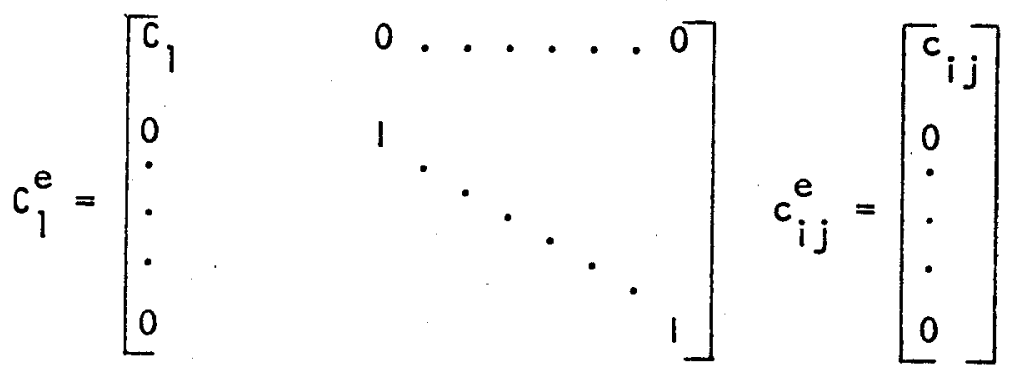

The system of (3.13) can be transformed into a similar. system using the transformation $T=\left[\begin{array}{ll}T_{1} & 0 \\ 0 & 1\end{array}\right]$ where $T_{1} \quad$ is an $n_{1}^{e} \times n_{1}^{e}$ matrix given by

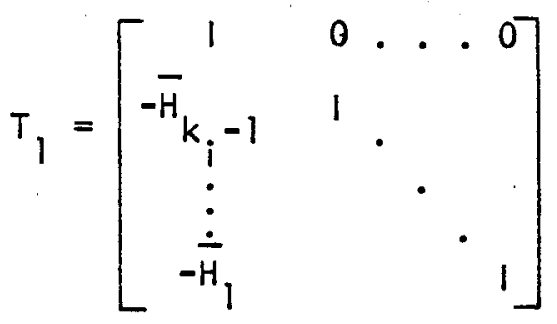

Let $\hat{A}_{1}^{e}, \hat{B}_{1}^{e}$, etc. represent the transformed system, then

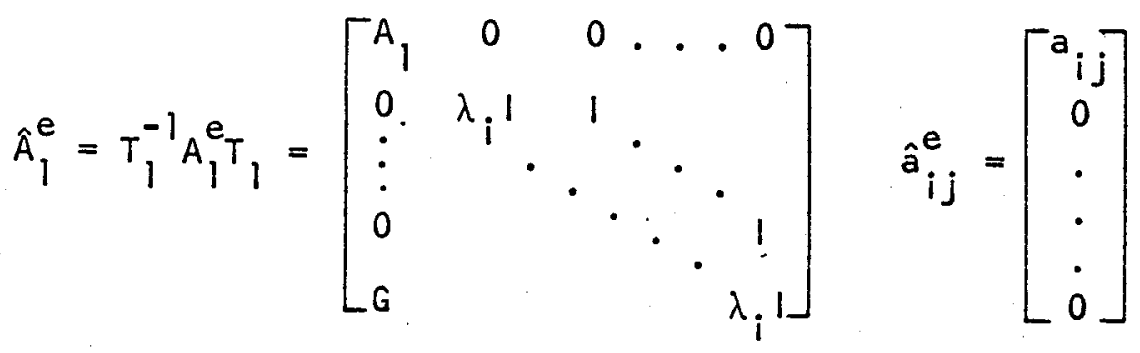

$$
\hat{B}_{1}^{e}=\left[\begin{array}{c}
B_{1} \\
0 \\
\vdots \\
0
\end{array}\right] \quad \hat{D}_{1}^{e}=\left[\begin{array}{lll}
D_{1} & 0 . . .0
\end{array}\right] a_{i j}^{e}=\left[d_{i j}\right]
$$


which is immediately recognized as the extension of equation (3.10). Since rank conditions, stabilizability, and detectability are invariant under similarity transformations, the extension of (3.14) obviously has the desired properties. Observe that this development used the variables defined by the matrix $G$ of (3.8) only for illustration. The same procedure applies to the variables defined by $\bar{D}$ of $(3.4)$, G' of (3.11), etc.

\section{PLANT PERTURBATIONS}

The coordinate system introduced in $\$ 2$ provides a convenient framework in which to introduce perturbations in the system maps $A$ and $B$. it is apparent that plant perturbations in $A$ are in effect only variations in $A_{1}$ and the columns of $A_{3}$ that are not identically zero and perturbations in the map $B$ only result in changes in $B_{1}$. Define

$$
A_{3}^{\prime} \equiv\left[\begin{array}{llll}
a_{11} & a_{12} & \cdots & a_{r q}
\end{array}\right]
$$

then perturbations in $(A, B)$ are perturbations in $\left(A_{1}, A_{3}^{1}, B_{1}\right)$ and we write these as

$$
\begin{aligned}
& \tilde{A}_{1} \equiv A_{1}+\delta \hat{A}_{1} /\|\hat{A}\| \\
& \tilde{A}_{3}^{\prime} \equiv A_{3}^{1}+\delta \hat{A}_{3} /\|\hat{A}\| \\
& \tilde{B}_{1} \equiv B_{1}+\delta \hat{B}_{1} /\|\hat{B}\|
\end{aligned}
$$

Using these ideas, arbitrary perturbations in $A$ and $B$ are defined by

Definition 4.1. RSFP is solvable for arbitrary perturbations in $A$ and $B$ if there exists a $\delta_{0}>0$ such that RSFP is solvable for $(\tilde{A}, \tilde{B}, C, D)$ with $0 \leq \delta<\delta_{0}$ and arbitrary $\hat{A}_{1}, \hat{A}_{3}^{1}, \hat{B}_{1}$. 
It is easily shown that the assumptions $(1.4)-(1.6)$ remain valid under arbitrary perturbations in $(A, B)$ and a simple applica tion of Corollary 2.1 proves that RSFP is never solvable for arbitrary perturbations in $(A, B)$. This result is presented as

Theorem 4.1

Assume (1.4) - (1.6) and (2.7) are true and RSFP is solvable for $(A, B, C, D)$. RSFP is never solvable for arbitrary perturbations in $(A, B)$.

Examples can be constructed for which certain types of perturbations are admissible. That is, for a particular $\left(\hat{A}_{1}, \hat{A}_{3}^{1}, \hat{B}_{1}\right)$ there exists a $\delta_{0}>0$ such that RSFP is solvable for $(\tilde{A}, \tilde{B}, C, D)$ with $0 \leq \delta<\delta_{0}$. It will often be the case that certain elements of $A_{1}, A_{3}^{\prime}$, or $B_{1}$ are known and not susceptible to error; for example, definitional relations like $\dot{\xi}_{1}=\xi_{2}$ fall into this category as do equations expressing well known physical laws. Thus, it is realistic and useful to characterize admissible perturbations which are defined by

Definition 4.2. Given $(A, B, C, D)$ and $\left(\hat{A}_{1}, \hat{A}_{3}, \hat{B}_{1}\right)$, a perturbation is admissible if there exists a $\delta_{0}>0$ such that RSFP is solvable for $(\tilde{A}, \tilde{B}, C, D)$ for $0 \leq \delta<\delta_{0}$

From Corollary 2.1 it is clear that constraints on $\left(\hat{A}_{1}, \hat{A}_{3}^{\prime}, \hat{B}_{1}\right)$ must be determined so that there exists a $\delta_{0}>0$ for which

$$
\operatorname{rank}\left[\begin{array}{cc}
\left(\tilde{A}_{1}-\lambda_{i} \mid\right) & \tilde{B}_{1} \\
D_{1} & 0
\end{array}\right]=\operatorname{rank}\left[\begin{array}{ccc}
\left(\tilde{A}_{1}-\lambda_{i}{ }^{1}\right. & \tilde{B}_{i} & \tilde{a}_{i j} \\
D_{i} & 0 & d_{i j}
\end{array}\right]=n_{i}
$$




$$
\begin{aligned}
& \operatorname{rank}\left[\begin{array}{ccc}
\left(\tilde{A}_{1}-\lambda_{i} 1\right)^{2} & \left(\tilde{A}_{1}-\lambda_{i}{ }^{1)} \tilde{B}_{1}\right. & \tilde{B}_{1} \\
D_{1} & 0 & 0
\end{array}\right]= \\
& \operatorname{rank}\left[\begin{array}{cccc}
\left(\tilde{A}_{1}-\lambda_{i} 1\right)^{2} & \left(\tilde{A}_{1}-\lambda_{i} 1\right) \tilde{B}_{1} & \tilde{B}_{1} & \tilde{a}_{i j} \\
D_{1} & 0 & 0 & 0
\end{array}\right]=n_{1}
\end{aligned}
$$

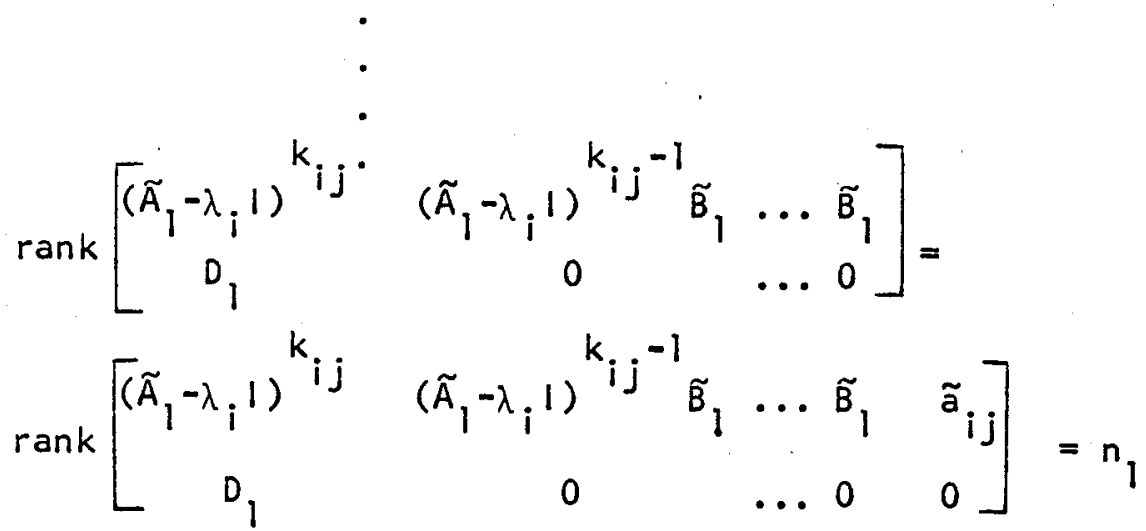

for $0 \leq \delta<\delta_{0}$ and each $i \in \bar{r}, j \in \bar{q}_{i}$.

A straightforward but tedious development provides a test by which the admissibility of a given $\left(\hat{A}_{1}, \hat{A}_{3}^{\prime}, \hat{B}_{1}\right)$ can be determined. This test is given in the following theorem:

Theorem 4.2

Assume $(1.4)-(1.6)$ and (2.7) are true, RSFP is solvable for $(A, B, C, D)$ and $F=\left[\begin{array}{ll}F_{1} & F_{2}\end{array}\right]$ is a solution. $\left(\hat{A}_{1}, \hat{A}_{3}^{1}, \hat{B}_{1}\right)$ is admissible if and only if for each $i \in \bar{r}$ and $j \in \bar{q}_{i}$

$$
D_{1}\left[\begin{array}{cc}
v_{l}^{t} & w_{l}^{t}
\end{array}\right]=0 \quad l=1,2, \ldots, t n,
$$

for $1 \leq t \leq k_{i j}$ where

$$
\begin{aligned}
& {\left[\begin{array}{ll}
v_{0}^{t} & w_{0}^{t}
\end{array}\right]=A_{\lambda_{i}}^{-t}\left[\begin{array}{ll}
B_{1} & a_{i j}
\end{array}\right] \quad\left[\begin{array}{ll}
v_{l}^{t} & w_{l}^{t}
\end{array}\right] \equiv 0 \text { if } l<0}
\end{aligned}
$$

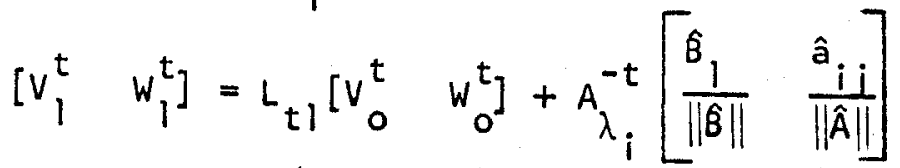

$$
\begin{aligned}
& {\left[\begin{array}{ll}
v_{l}^{t} & w_{l}^{t}
\end{array}\right]=\sum_{s=1}^{t} L_{t s}\left[\begin{array}{ll}
t & w_{l-s}^{t}
\end{array}\right] \quad l \geq 2}
\end{aligned}
$$




$$
\begin{array}{r}
A_{\lambda_{i}} \equiv\left(A_{1}+B_{1} F_{1}-\lambda_{i}{ }^{\prime}\right) \text { and the } L_{t s} \frac{\text { are defined by }}{t} \\
\left(\tilde{A}_{1}+\tilde{B}_{1} F_{1}-\lambda_{i}\right)^{t}=\left(A_{1}+B_{1} F_{1}-\lambda_{i} 1\right)^{t}\left(1-\sum_{s=1}^{t} \delta^{s} L_{t s}\right) .
\end{array}
$$

Theorem 4.2 clearly reveals the technique to be followed when certain parameters of $(A, B)$ are susceptible to variations. RSFP is solved and an $F$ is obtained. Then the perturbation of concern $\left(\hat{A}_{1}, \hat{A}_{3}^{\prime}, \hat{B}_{1}\right)$ is tested via (4.4) to see whether or not it is admissible.

\section{CONCLUSION}

In this paper we have formulated and solved a general linear servomechanism problem using the approach developed in [1] and [6]. We have shown conditions under which it is possible to design a robust control system so that tracking and internal stability are maintained in the case of perturbations in the controller parameters. We have shown that robust solutions do not exist for arbitrary perturbations in plant parameters and have defined a class of admissible plant perturbations for which our problem is solvable. In general, when RSFP is not solvable without extension, the additional dynamics introduced by the extension must duplicate exactly a partial (in general) description of the class of exogenous signals. This situation has been referred to in [4] as the "internal model principle" and is of fundamental importance in the theory of linear servomechanisms. 


\section{REFERENCES}

1. J.B. Pearson, R.W. Shields, and P.W. Staats, Jr., "Robust solutions to linear multivariable control problems," Proceedings: 1974 Joint Automatic Control Conference, University of Texas, June 18-21, 1974, pp. 172176, to appear IEEE Trans. A-C, Oct. 1974.

2. W.M. Wonham and J.B. Pearson, "Regulation and internal stabilization in linear multivariable systems," SIAM J. Control, vol. 12, no. 1, Feb. 1974, pp. 5-18.

3. E.J. Davison, "The output control of linear multivariable systems with unmeasurable arbitrary disturbances," IEEE Trans. A-C, vol. AC-17, Oct. 1972 , pp. $621-630$.

4. B. Francis, O.A. Sebakby and W.M. Wonham, "Synthesis of Multivariable Regulators: The Internal Model Principle," Control Systems Report No. 7312, Department of Electrical Engineering, University of Toronto, Oct. 1973.

5. P.R. Halmos, Finite-Dimensional Vector Spaces, D. Van Nostrand Co., Inc., New York, N.Y., 1958, Pp. 150-152.

6. J.B. Pearson and P.W. Staats, Jr., "Robust controllers for linear regulators," IEEE Trans. A-C, vol. AC-19, no. 3, June 1974, pp. 231-234.

7. D.M. Young, Iterative Solution of Large Linear Systems, Academic Press, New York, N.Y., 1971, p. 15. 


\section{APPENDIX}

Two Lemmas are necessary for the proof of Theorem 2.1.

Lemma 2.1

Given $A: X \rightarrow X, B: U \rightarrow X$ and $F: X \rightarrow U$, such that $\sigma(A+B F) \subset c^{-}$. There exists $\varepsilon_{0}>0$ such that $\sigma(A+B \tilde{F}) \subset c^{-}$for all $\hat{F}$ and $0 \leq \epsilon<\epsilon_{0}$.

The proof follows by direct application of Theorem 1.10 in [7] and will not be reproduced here.

\section{Lemma 2.2}

Assume $(1.4)-(1.6)$ are true, RPIS is solvable and $F: x \rightarrow U$ is a solution to RPIS. Then

$$
B \subset\left(A+B F-\lambda_{i}\right)^{j} \operatorname{Ker} D
$$

if and only if

$$
B_{1} \subset\left(A_{1}+B_{1} F_{1}-\lambda_{i}\right)^{j} \operatorname{Ker} D_{1}
$$

where $\lambda_{i} \in \sigma\left(A_{2}\right)$ and $j$ is a positive integer. $A_{1}, B_{1}, D_{1}$ are defined in (1.3) and $F_{1}$ is a partition of $F=\left[\begin{array}{lll}F_{1} & F_{2}\end{array}\right]$.

In order to present the basic ideas in a simple manner, the following proofs are presented under the assumption that the minimal polynomial of $A_{2}$ has only real roots. These proofs are easily modified for complex roots using the concept of complexification as discussed, e.g., by Halmos [5].

\section{Proof of Lemma 2.2}

(suff) Assume $B_{1} \subset\left(A_{1}+B_{1} F_{1}-\lambda_{i}\right)^{j} \operatorname{Ker} D_{1} \cdot C l e a r l y \quad \operatorname{Ker} D_{1} \subset \operatorname{Ker} D$ and using (1.2) 
$B=B_{1} \subset\left(A_{1}+B_{1} F_{1}-\lambda_{i}\right)^{j} \operatorname{Ker} D_{1}=\left(A+B F-\lambda_{i}\right)^{j} \operatorname{Ker} D_{1} \subset\left(A+B F-\lambda_{i}\right)^{j} \operatorname{Ker} D$

(nec) Since $F$ is a solution to RPIS, $F$ stabilizes the plant. Thus, $x^{+}(A+B F) \cap x_{1}=0$ and $d\left(x^{+}(A+B F)\right)=d\left(x_{2}\right)$. Let $\mathcal{L}=\operatorname{Ker} D_{1} \subset x_{1}$, then $\mathcal{L} \cap \mathcal{X}^{+}(A+B F)=0$ and $\mathcal{L} \oplus \mathcal{X}^{+}(A+B F) \subset \operatorname{Ker} D$. By assumption $D_{1}$ is epic, thus $D$ is epic and $d(d)=d\left(Q_{1}\right)$. Then $d(\mathcal{L})=d\left(X_{1}\right)-d\left(D_{1}\right)=d\left(X_{1}\right)-d(D)$ and $d\left(\mathcal{L} \oplus X^{+}(A+B F)\right)=d\left(X_{1}\right)-d(Q)+d\left(X_{2}\right)=d(X)-d(D)=d(\operatorname{Ker} D)$. So, $\mathcal{L} \oplus \mathcal{X}^{+}(A+B F)=\operatorname{Ker} D$.

The subspace $\chi^{+}(A+B F)$ is $(A+B F)$-invariant. Thus for any real $\lambda_{i}$ and integer $j,\left(A+B F-\lambda_{i}\right) x^{j}(A+B F) \subset x^{+}(A+B F)$. Now assume that $B \subset\left(A+B F-\lambda_{j}\right) j_{\text {Ker }} D$. since $B=B_{1} \subset x_{1}$,

$$
\begin{aligned}
B_{1} & \subset\left[\left(A+B F-\lambda_{i}\right)^{j} \operatorname{ker} D\right] \cap x_{1}=\left[\left(A+B F-\lambda_{i}\right)^{j} \mathcal{L}+\left(A+B F-\lambda_{i}\right)^{j} x^{+}(A+B F)\right] \cap x_{1} \\
& =\left(A+B F-\lambda_{i}\right)^{j} \mathcal{L}+\left[\left(A+B F-\lambda_{i}\right)^{j} x^{+}(A+B F)\right] \cap x_{i} \\
& \subset\left(A_{1}+B_{1} F_{1}-\lambda_{i}\right)^{j} \operatorname{ker} D_{1}+x^{+}(A+B F) \cap x_{1} \\
& =\left(A,+B F_{1}-\lambda_{i}\right)^{j} \operatorname{ker} D_{1}
\end{aligned}
$$

Q.E.D.

The proof of Theorem 2.1 utilizes the structure inherent in the Jordan decomposition of a space relative to a given map. In particular, the Jordan. decomposition technique can be used to decompose $x_{2}$ relative to $A_{2}$ into a direct sum of cyclic subspaces. As discussed in $\delta 2$, let $\sigma\left(A_{2}\right)=\left\{\lambda_{1}, \ldots, \lambda_{r}\right\}$ and define

$$
q_{i} \equiv d\left(\operatorname{Ker}\left(A_{2}-\lambda_{i}\right)\right)
$$

Then $q_{i}$ is the number of cyclic subspaces of $x_{2}$ related to $\lambda_{i}$ in this decomposition. These subspaces are written as $x_{2 i}^{1}, \ldots, x_{2}{ }^{q}$ and their dimen- 
sions are

$$
k_{i j} \equiv d\left(x_{2 i}^{j}\right)
$$

Furthermore, the subspaces can be indexed so that $k_{i j} \geq \ldots \geq k_{i q}$. Note that this yields $k_{i l}=k_{i}$.

Assume that RPIS is solvable and $F$ is a solution. In the same manner as above, $X$ can be decomposed relative to (A+BF). Since $F$ solves RPIS, F stabilizes the plant and $\sigma\left(A_{1}+B_{1} F_{l}\right) \subset C^{-}$. This implies $\sigma(A+B F) \cap C^{+}=$ $\sigma\left(A_{2}\right)$ and $d\left(\operatorname{Ker}\left(A+B F-\lambda_{i}\right)\right)=q_{i}$ for each $\lambda_{i} \in \sigma(A+B F) \cap c^{+}$. The cyclic subspaces related to $\lambda_{i}$ are written as $x_{i}, \ldots, x_{i} q_{i}$ and can be indexed so that

$$
k_{i j}=d\left(x_{i}^{j}\right)
$$

Now consider $x_{i}^{l}$ and $x_{2 i}^{l}$ for some $i \in \bar{r}$ where $r$ is the number of distinct eigenvalues of $A_{2}$. Since $x_{2 i}^{\prime} \subset x_{2}$ is a cyclic subspace, there is a vector $x_{2 k_{i}} \in x_{2}$ that generates $x_{2 i}^{\prime}$. That is $x_{2 i}^{\prime}=\left\{x_{21}, \ldots, x_{2 k_{i}}\right\}$ where

$$
\left(A_{2}-\lambda_{i}\right)^{k_{i}-t} x_{2 k_{i}}=x_{2 t} \quad t \in \bar{k}_{i}
$$

Since $d\left(x_{i}^{l}\right)=d\left(x_{2 i}^{i}\right)$ and $x_{i}^{l} \cap x_{1}=0$ there is a vector $x_{1 k_{i}} \in x_{1}$ such that $x_{k_{i}}=x_{1 k_{i}} \oplus x_{2 k_{i}}$ is the generator for the cyclic subspace $x_{i}^{l}$. Hence $x_{i}^{\prime}=\left\{x_{1}, \ldots, x_{k_{i}}\right\}$ where

$$
\left(A+B F-\lambda_{i}\right)^{k_{i}-t} x_{k_{i}}=x_{t} \quad t \in \bar{k}_{i}
$$

Observe that the $x_{t}$ can be written as

$$
x_{t}=x_{1 t} \oplus x_{2 t}
$$

where $x_{2 t}$ and $x_{1 t}$ are defined by $(A .4)$ and (A.5). Finally note that 
$x_{k_{i}}$ being a generator implies $\left(A+B F-\lambda_{i}\right)^{k} x_{k_{i}}=0$ and using (A.5) it follows that $\left(A+B F-\lambda_{i}\right){ }^{k} x_{t}=0$ for $t \in \bar{k}_{i}$. Thus

$$
x_{i}^{\prime} \subset x^{+}(A+B F) \subset \operatorname{Ker} D
$$

Proof of Theorem 2.1

(nec) Assume that RSFP has a solution. Thus there exists an $F: x \rightarrow U$ and an $\varepsilon_{0}>0$ such that $\eta \subset \operatorname{ker} F$ and

$$
\begin{aligned}
& x^{+}(A+B \tilde{F}) \subset \operatorname{ker} D \\
& x^{+}(A+B \tilde{F}) \cap\langle A \mid B\rangle=0
\end{aligned}
$$

for all admissible $\tilde{F} \in N\left(F, \epsilon_{0}\right)$. Since $\epsilon=0$ clearly results in an admissible perturbation, (A.8) implies that RPIS is solvable and $F$ is a solution. Consider some $\hat{F}$ and $\varepsilon_{1}$ such that $\eta \subset \operatorname{ker} \hat{F}$ and $0<\epsilon_{1}<\varepsilon_{0}$, then $\tilde{F}=F+\varepsilon_{1} \hat{F} /\|\hat{F}\|$ is admissible. Thus RPIS is solvable for $\tilde{F}$ and $\chi$ can be decomposed relative to $(A+B \tilde{F})$. The same development as before leads to a cyclic subspace $\tilde{x}_{i}^{l} \subset x$ that is generated by a vector $\tilde{x}_{k_{i}}=\tilde{x}_{1 k_{i}} \oplus x_{2 k_{i}}$ and spanned by basis vectors defined by

$$
\left(A+B \tilde{F}-\lambda_{i}\right)^{k_{i}-t} \tilde{x}_{k_{i}}=\tilde{x}_{t} \quad t \in \bar{k}_{i}
$$

In addition

$$
\tilde{z}_{i}^{\top} \subset \operatorname{Ker} D
$$

and the $\tilde{x}_{t}$ can be written as

$$
\tilde{x}_{t}=\tilde{x}_{1 t} \oplus x_{2 t}
$$

For $t=1$, equations $(A .9)$ and $(A .10)$ give 


$$
\begin{aligned}
& \left(A+B \tilde{F}-\lambda_{i}\right) \tilde{x}_{1}=0 \\
& D \tilde{x}_{1}=0
\end{aligned}
$$

And from $(A .5)-(A .7)$

$$
\begin{aligned}
& \left(A+B F-\lambda_{i}\right) x_{1}=0 \\
& D x_{1}=0
\end{aligned}
$$

Subtracting the above equations and defining $\hat{x}_{11} \equiv \tilde{x}_{11}-x_{11}$ and $\epsilon_{1}^{\prime} \equiv \epsilon_{1} /\|\hat{F}\|$ yields

$$
\begin{aligned}
& \left(A_{1}+B_{1} F_{1}-\lambda_{1}\right) \hat{x}_{11}+\varepsilon_{1}^{\prime} B_{1}\left(\hat{F}_{1} \tilde{x}_{11}+\hat{F}_{2} x_{21}\right)=0 \\
& D_{1} \hat{x}_{11}=0
\end{aligned}
$$

Since $n \subset x_{1}, \hat{F}_{1}=0$ yields an admissible perturbation for $0<\varepsilon_{1}<\varepsilon_{0}$. Thus

$$
\begin{aligned}
& \left(A_{1}+B_{1} F_{1}-\lambda_{1}\right) \hat{x}_{11}=-\epsilon_{1}^{1} B_{1} \hat{F}_{2} x_{21} \\
& D_{1} \hat{x}_{11}=0
\end{aligned}
$$

Note that $x_{21} \neq 0$ and admissibility restrictions affect only $\hat{F}_{j}$. Thus (A.12) must have a solution $\hat{x}_{11} \in x_{1}$ for any $\hat{F}_{2}$. This implies

$$
B_{1} \subset\left(A_{1}+B_{1} F_{1}-\lambda_{i}\right) \operatorname{Ker} D_{1}
$$

and by Lemma 2.2

$$
B \subset\left(A+B F-\lambda_{i}\right) \operatorname{Ker} D
$$

Now consider $t$ such that $l<t \leq k_{i}$ and assume that

$$
B_{1} \subset\left(A_{1}+B_{1} F_{1}-\lambda_{i}\right)^{j} \operatorname{Ker} D_{1} \quad j \in \overline{t-1}
$$

From equations $(A .9)-(A .11)$ and the relations of (1.2)

$$
\begin{aligned}
& \left(A_{1}+B_{1} \tilde{F}_{1}-\lambda_{i}\right) \tilde{x}_{1 t}+\left(A_{3}+B_{1} \tilde{F}_{2}\right) x_{2 t}=\tilde{x}_{1 t-1} \\
& D_{1} \tilde{x}_{1 t}+D_{2} x_{2 t}=0
\end{aligned}
$$


Similarly

$$
\begin{aligned}
& \left(A_{1}+B_{1} F_{1}-\lambda_{i}\right) x_{i t}+\left(A_{3}+B_{1} F_{2}\right) x_{2 t}=x_{1 t-1} \\
& D_{1} x_{1 t}+D_{2} x_{2 t}=0
\end{aligned}
$$

and upon setting $\hat{F}_{1}=0$, defining $\hat{x}_{1 t} \equiv \tilde{x}_{1 t}-x_{1 t}$, and subtracting the above equations

$$
\begin{aligned}
& \left(A_{1}+B_{1} F_{1}-\lambda_{i}\right) \hat{x}_{1 t}=-\varepsilon_{1} B_{1} F_{2} x_{2 t}+\hat{x}_{1 t-1} \\
& D_{1} \hat{x}_{1 t}=0
\end{aligned}
$$

The map $\left(A_{1}+B_{1} F_{1}-\lambda_{i}\right)$ operates on the first equation of (A.14) to give

$$
\left(A_{1}+B_{1} F_{1}-\lambda_{i}\right)^{2} \hat{x}_{1 t}=-\varepsilon !\left(A_{1}+B_{1} F_{1}-\lambda_{i}\right) B_{1} \hat{F}_{2} x_{2 t}-\varepsilon_{1}^{\prime} B_{1} \hat{F}_{2} x_{2 t-1}+\hat{x}_{1 t-2}
$$

Repeated application of $\left(A_{1}+B_{1} F_{1}-\lambda_{i}\right)$ leads to

$$
\begin{aligned}
\left(A_{1}+B_{1} F_{1}-\lambda_{i}\right)^{t_{x_{1}}=} & -\varepsilon_{1}^{\prime}\left(A_{1}+B_{1} F_{1}-\lambda_{i}\right)^{t-1_{B} F_{2} x_{2 t}} \\
& \vdots \\
& -\epsilon_{1}^{\prime}\left(A_{1}+B_{1} F_{1}-\lambda_{i}\right) B_{1} \hat{F}_{2} x_{22} \\
& -\epsilon_{1}^{\prime} B_{1} F_{2} x_{21} \\
D_{1} \hat{x}_{1 t}=0 &
\end{aligned}
$$

But from $(A .13),\left(A_{1}+B_{1} F_{1}-\lambda_{i}\right)^{j_{B}} \subset\left(A_{1}+B_{1} F_{1}-\lambda_{i}\right)^{t} \operatorname{ker} D_{1}$ for $j \in \overline{t-1}$. Thus there exist $x_{i s}^{i} \in \operatorname{Ker} D_{1}$ such that $\epsilon_{1}^{\prime}\left(A_{1}+B_{1} F_{1}-\lambda_{i}\right)^{t-s_{B}} F_{2} x_{2 t-s+1}=$ $\left(A_{1}+B_{1} F_{1}-\lambda_{i}\right)^{t} x_{i s}^{1}$ for $s \in \overline{t-1}$. Let $x_{1 t}^{*}=\hat{x}_{1 t}+x_{11}^{1}+\ldots+x_{1 t-1}^{1}$, then

(A.15) becomes

$$
\begin{aligned}
& \left(A_{1}+B_{1} F_{1}-\lambda_{i}\right)^{t} x_{1 t}^{*}=-\varepsilon_{1}^{\prime} B_{1} F_{2} x_{21} \\
& D_{1} x_{1 t}^{*}=0
\end{aligned}
$$

implying

$$
B_{1} \subset\left(A_{1}+B_{1} F_{1}-\lambda_{i}\right)^{t} \operatorname{Ker} D_{1} \quad t \in \bar{k}_{i}
$$


Thus by Lemma 2.2

$$
B \subset \bigcap_{j=1}^{k}\left(A+B F-\lambda_{i}\right)^{j} \operatorname{Ker} D
$$

for each $\lambda_{i} \in \sigma\left(A_{2}\right)$.

(suff) Assume that RPIS is solvable, $F$ is a solution and (2.4) is true. By Lemma 2.1 an $\epsilon_{0}>0$ can be selected such that $x^{+}(A+B \tilde{F}) \cap\langle A \mid B\rangle=0$ for all admissible $\tilde{F}$. Upon choosing any admissible $\tilde{F}$, the procedure of the necessity of proof can essentially be applied in reverse to define the subspaces $\tilde{X}_{i}^{j}$ with the properties

$$
\begin{aligned}
& x_{i}^{j} \subset x^{+}(A+B \tilde{F}) \\
& x_{i}^{j} \subset \operatorname{Ker} D
\end{aligned}
$$

for each $i \in \bar{r}, j \in \bar{q}_{i}$. Then

$$
x^{+}(A+B \tilde{F})=\bigoplus_{i=1}^{r}\left[\oplus_{j=1}^{q} \tilde{x}_{i}^{j}\right] \subset \operatorname{Ker} D
$$

$$
\text { Q.E.D. }
$$

\section{Proof of Corollary 2.1}

(nec) Assume that RSFP has a solution. The feedback map $F: X \rightarrow U$ that solves RSFP has a matrix representation $F=\left[\begin{array}{lll}F_{1} & F_{2}\end{array}\right]$ and $F_{2}$ can be partitioned consistent with the partitions of $A_{3}$ and $D_{2}$ as

$$
F_{2}=\left[\begin{array}{llll}
F_{11} & F_{12} & \cdots & F_{r q}
\end{array}\right]
$$

where $F_{i j}$ is an $m \times k_{i j}$ matrix. Since $F$ solves RPIS, the plant is sta- bilized and $\sigma\left(A_{1}+B_{1} F_{1}\right) \subset C^{-}$. Thus there exist $n_{1} \times k_{i j}$ matrices $v_{i j}$ such. that 


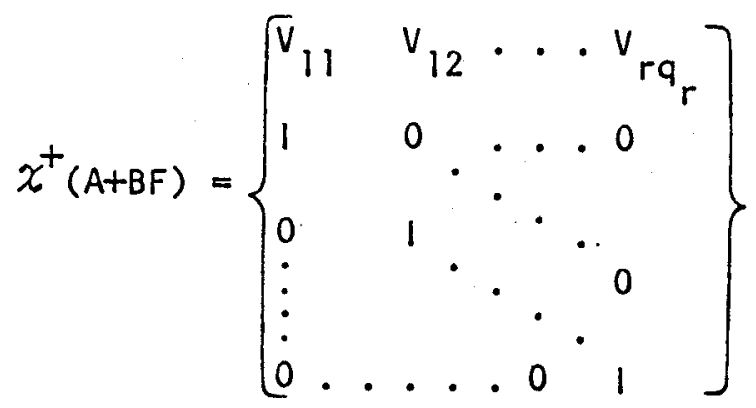

Recall that $(A+B F) x^{+}(A+B F) \subset x^{+}(A+B F)$ and $x^{+}(A+B F) \subset \operatorname{ker} D$. So for each $i, j$ such that $i \in \bar{r}$ and $j \in \bar{q}_{i}$, the following matrix equations are true:

$$
\begin{aligned}
& \left(A_{1}+B_{1} F_{1}\right) V_{i j}+\left(A_{i j}+B_{1} F_{i j}\right)=V_{i j} J_{i j} \\
& D_{1} V_{i j}+D_{i j}=0
\end{aligned}
$$

where $J_{i j}$ is the $i j^{\text {th }}$ Jordan Block of $A_{2}$. The matrices $V_{i j}$ and $F_{i j}$ can be written as $v_{i j}=\left[\begin{array}{llll}v_{1} & v_{2} & \cdots & v_{k_{i j}}\end{array}\right] \quad F_{i j}=\left[\begin{array}{llll}f_{i} & f_{2} & \cdots & f_{k_{i j}}\end{array}\right]$. Then by $(A .16)$

$$
\begin{aligned}
& \left(A_{1}+B_{1} F_{1}\right) v_{1}+a_{i j}+B_{1} f_{1}=\lambda_{i} v_{1} \\
& D_{1} v_{1}+d_{i j}=0
\end{aligned}
$$

which can be written as

$$
\begin{aligned}
& \left(A_{1}+B_{1} F_{1}-\lambda_{i}\right) v_{1}+B_{1} f_{1}+a_{i j}=0 \\
& D_{1} v_{1}+d_{i j}=0
\end{aligned}
$$

This implies

$$
\operatorname{rank}\left[\begin{array}{cc}
\left(A_{1}+B_{1} F_{1}-\lambda_{i}\right) & B_{1} \\
D_{1} & 0
\end{array}\right]=\operatorname{rank}\left[\begin{array}{ccc}
\left(A_{1}+B_{1} F_{1}-\lambda_{i}\right) & B_{l} & a_{i j} \\
D_{l} & 0 & d_{i j}
\end{array}\right]
$$

From equation (2.4)

$$
\operatorname{rank}\left[\begin{array}{c}
\left(A_{1}+B_{1} F_{1}-\lambda_{i}\right) \\
D_{1}
\end{array}\right]=\operatorname{rank}\left[\begin{array}{cc}
\left(A_{1}+B_{1} F_{1}-\lambda_{i}\right) & B_{1} \\
D_{1} & 0
\end{array}\right]
$$

and since $\left(A_{1}+B_{1} F_{1}-\lambda_{i}\right)$ is invertible, equations (A.17) and $(A .18)$ combine 
to give

$$
\operatorname{rank}\left[\begin{array}{cc}
\left(A_{1}-\lambda_{i}\right) & B_{1} \\
D_{1} & 0
\end{array}\right]=\operatorname{rank}\left[\begin{array}{ccc}
\left(A_{1}-\lambda_{i}\right) & B_{1} & a_{i j} \\
D_{1} & 0 & d_{i j}
\end{array}\right]=n_{1}
$$

Looking at (A.16) again, it is clear that

$$
\begin{aligned}
& \left(A_{1}+B_{1} F_{1}-\lambda_{i}\right) v_{2}+B_{1} f_{2}=v_{1}+\lambda_{i} v_{2} \\
& D_{1} v_{2}=0
\end{aligned}
$$

Rewriting and multiplying by $\left(A_{1}+B_{1} F_{1}-\lambda_{i}\right)$ gives

$$
\begin{aligned}
\left(A_{1}+B_{1} F_{1}-\lambda_{i}\right)^{2} v_{2}+\left(A_{1}+B_{1} F_{1}-\lambda_{i}\right) B_{1} f_{2} & =\left(A_{1}+B_{1} F_{1}-\lambda_{i}\right) v_{1} \\
& =-a_{i j}-B_{l} f_{1}
\end{aligned}
$$

Thus

$$
\begin{aligned}
& \left(A_{1}+B_{1} F_{1}-\lambda_{i}\right)^{2} v_{2}+\left(A_{1}+B_{1} F_{1}-\lambda_{i}\right) B_{1} f_{2}+B_{1} f_{1}+a_{i j}=0 \\
& D_{1} v_{2}=0
\end{aligned}
$$

which implies

$$
\begin{aligned}
& \operatorname{rank}\left[\begin{array}{ccc}
\left(A_{1}+B_{1} F_{1}-\lambda_{i}\right)^{2} & \left(A_{1}+B_{1} F_{1}-\lambda_{i}\right) B_{1} & B_{l} \\
D_{1} & 0 & 0
\end{array}\right] \\
= & \operatorname{rank}\left[\begin{array}{cccc}
\left(A_{1}+B_{1} F_{1}-\lambda_{i}\right)^{2} & \left(A_{1}+B_{1} F_{1}-\lambda_{i}\right) B_{1} & B_{1} & a_{i j} \\
D_{l} & 0 & 0 & 0
\end{array}\right]
\end{aligned}
$$

From equation (2.4) $B_{1}+\left(A_{1}+B_{1} F_{1}-\lambda_{i}\right) B_{1} \subset\left(A_{1}+B_{1} F_{1}-\lambda_{i}\right)^{2} \operatorname{Ker} D_{1}$ or $\operatorname{rank}\left[\begin{array}{c}\left(A_{1}+B_{1} F_{1}-\lambda_{i}\right)^{2} \\ D_{1}\end{array}\right]=\operatorname{rank}\left[\begin{array}{ccc}\left(A_{1}+B_{1} F_{1}-\lambda_{i}\right)^{2} & \left(A_{1}+B_{1} F_{1}-\lambda_{i}\right) B_{1} & B_{1} \\ D_{1} & 0 & 0\end{array}\right]$

Equations (A.19) and (A.20) combine to give

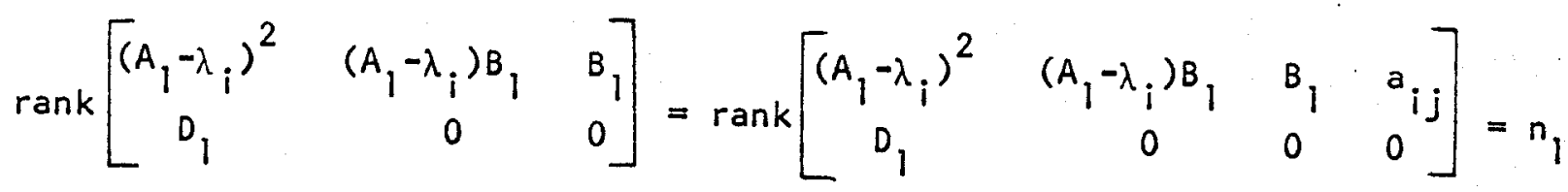


Since $k_{i j} \leq k_{i l}$, this procedure can clearly be continued to show the validity of $(2.8)$

(suff) Assume (2.8) is true. By (1.4) and (1.6), an $F_{1}$ can be selected such that $\sigma\left(A_{1}+B_{1} F_{1}\right) \subset C^{-}$and $\eta \subset \operatorname{ker} F$ for any $F_{2}$.

Pick any $i, j$ such that $i \in \bar{r}$ and $j \in \bar{q}_{\left[\left(A_{1}+B_{1} F_{1}-\lambda_{i}\right)\right.}$. Since $\lambda_{i} \in C^{\dot{ }}$, $\left(A_{1}+B_{1} F_{1}-\lambda_{i}\right)$ is invertible and $\operatorname{rank}\left[\begin{array}{c}\left.A_{1}+B_{1} F_{1}-\lambda_{i}\right) \\ D_{1}\end{array}\right]=n_{1}$, for any integer

$t$. Then from the first equation (2.8)

$$
\operatorname{rank}\left[\begin{array}{c}
\left(A_{1}+B_{1} F_{1}-\lambda_{i}\right) \\
D_{1}
\end{array}\right]=\operatorname{rank}\left[\begin{array}{cc}
\left(A_{1}+B_{1} F_{1}-\lambda_{i}\right) & B_{1} \\
D_{1} & 0
\end{array}\right]
$$

which implies $B_{1} \subset\left(A_{1}+B_{1} F_{1}-\lambda_{i}\right) \operatorname{Ker} D_{1}$. Equation (2.8) also implies

$$
\operatorname{rank}\left[\begin{array}{c}
\left(A_{1}+B_{1} F_{1}-\lambda_{i}\right) \\
D_{1}
\end{array}\right]=n_{1}=\operatorname{rank}\left[\begin{array}{ccc}
\left(A_{1}+B_{1} F_{1}-\lambda_{i}\right) & B_{1} & a_{i j} \\
D_{1} & 0 & d_{i j}
\end{array}\right]
$$

Hence, for any $f_{1}$ there exists a matrix $v_{1}$ such that

$$
\begin{aligned}
& \left(A_{1}+B_{1} F_{1}-\lambda_{i}\right) v_{1}+a_{i j}+B_{1} f_{1}=0 \\
& D_{1} v_{1}+d_{i j}=0
\end{aligned}
$$

From the second equation of (2.8) it follows that

$$
\operatorname{rank}\left[\begin{array}{c}
\left(A_{1}+B_{1} F_{1}-\lambda_{i}\right)^{2} \\
D_{1}
\end{array}\right]=\operatorname{rank}\left[\begin{array}{ccc}
\left(A_{1}+B_{1} F_{1}-\lambda_{i}\right)^{2} & \left(A_{1}+B_{1} F_{1}-\lambda_{i}\right) B_{1} & B_{1} \\
D_{1} & 0 & 0
\end{array}\right]
$$

and thus

$$
B_{1} \subset\left(A_{1}+B_{1} F_{1}-\lambda_{i}\right)^{2} \operatorname{Ker} D_{1} \text {. }
$$

Equation (2.8) also implies 


$$
\operatorname{rank}\left[\begin{array}{c}
\left(A_{1}+B_{1} F_{1}-\lambda_{i}\right)^{2} \\
D_{1}
\end{array}\right]=n_{1}=\operatorname{rank}\left[\begin{array}{cccc}
\left(A_{1}+B_{1} F_{1}-\lambda_{i}\right)^{2} & \left(A_{1}+B_{1} F_{1}-\lambda_{i}\right) B_{1} & B_{1} & a_{i j} \\
D_{1} & 0 & 0 & 0
\end{array}\right]
$$

: So for the $f_{1}$ selected above and any $f_{2}$ there exists a matrix $v_{2}$ such that

$$
\begin{aligned}
& \left(A_{1}+B_{1} F_{1}-\lambda_{i}\right)^{2} v_{2}+\left(A_{1}+B_{1} F_{1}-\lambda_{i}\right) B_{1} f_{2}+B_{1} f_{1}+a_{i j}=0 \\
& D_{1} v_{2}=0
\end{aligned}
$$

and multiplying by $\left(A_{1}+B_{1} F_{1}-\lambda_{i}\right)^{-1}$ gives

$$
\begin{aligned}
& \left(A_{1}+B_{1} F_{1}-\lambda_{i}\right) v_{2}+B_{1} f_{2}=v_{1} \\
& D_{1} v_{2}=0
\end{aligned}
$$

Continuing in this manner clearly defines a matrix $v_{i j}=\left[\begin{array}{llll}v_{1} & v_{2} & \cdots & v_{k_{i j}}\end{array}\right]$ for any $F_{i j}=\left[\begin{array}{lllll}f_{1} & f_{2} & \ldots & f_{k_{i j}}\end{array}\right]$ selected such that

$$
\begin{aligned}
& \left(A_{1}+B_{1} F_{1}\right) V_{i j}+A_{i j}+B_{1} F_{i j}=V_{i j} J_{i j} \\
& D_{1} V_{i j}+D_{i j}=0
\end{aligned}
$$

and

$$
B_{1} \subset\left(A_{1}+B_{1} F_{1}-\lambda_{i}\right)^{t} \operatorname{Ker} D_{1} \quad t \in \bar{k}_{i j}
$$

Following this procedure for each $i \in \bar{r}$ and $j \in \bar{q}_{i}$. Defines matrices $V_{11}, V_{12}, \ldots, v_{r q_{r}}$ for any $F_{11}, F_{12}, \ldots, F_{r q_{r}}$ selected such that if $v \subset x$ is defined by

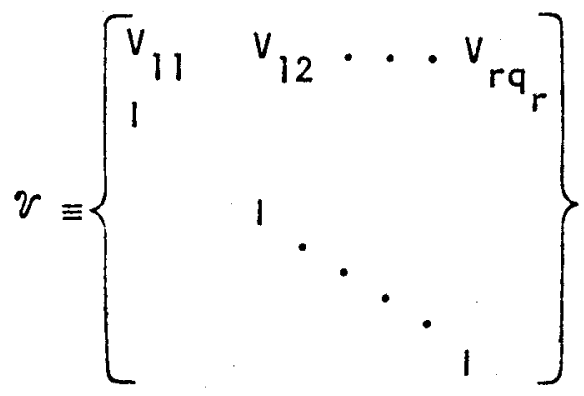

and $F_{2} \equiv\left[\begin{array}{lllll}F_{11} & F_{12} & \cdots & F_{r q}\end{array}\right]$, then (A.23) implies that $(A+B F) v \subset v$ and 
$v \subset \operatorname{ker} D$. Observe that since $\sigma\left(A_{1}+B_{1} F_{1}\right) \subset C^{-}, d(\gamma)=d\left(\chi^{+}(A+B F)\right)$ and from (A.23) $v \subset \chi^{+}(A+B F)$. Thus $F=\left[\begin{array}{ll}F_{1} & F_{2}\end{array}\right]$ solves RPIS. Finally, Lemma 2.2 can be applied to $(A .24)$ to give (2.4).

Q.E.D.

Proof of Theorem 4.1

Assume the contrary, i.e., there exists a $\delta_{0}>0$ such that RSFP is solvable for $(\tilde{A}, \widetilde{B}, C, D)$ for $0 \leq \delta<\delta_{0}$ with $\hat{A}_{1}, \hat{A}_{3}^{\prime}, \hat{B}_{1}$ arbitrary. Since $\hat{A}$, and $\hat{B}_{1}$ are arbitrary, we can pick $\hat{A}_{1} \equiv 0, \hat{B}_{1} \equiv 0$ and from Corollary 2.1

$$
\operatorname{rank}\left[\begin{array}{ccc}
\left(A_{1}-\lambda_{i} l\right) & B_{1} & \tilde{a}_{i j} \\
D_{1} & 0 & d_{i j}
\end{array}\right]=n_{1}
$$

for $0 \leq \delta<\delta_{0}$. By assumption $\left(A_{1}, B_{1}\right)$ is stabilizable. Thus there is an $F_{1}$ such that $\sigma\left(A_{1}+B_{1} F_{1}\right) \subset C^{-}$and from (A.25)

$$
\operatorname{rank}\left[\begin{array}{c}
\left(A_{1}+B_{1} F_{1}-\lambda_{i} 1\right) \\
D_{l}
\end{array}\right]=n_{1}=\operatorname{rank}\left[\begin{array}{ccc}
\left(A_{1}+B_{1} F_{1}-\lambda_{i}{ }^{\prime}\right) & B_{1} & a_{i j} \\
D_{1} & 0 & d_{i j}
\end{array}\right]
$$

for $0 \leq \delta<\delta_{0}$ and $\hat{a}_{i j}$ arbitrary. Hence a vector $\tilde{v}$ exists such that

$$
\begin{aligned}
& \left(A_{1}+B_{1} F_{1}-\lambda_{i}\right) \tilde{v}=\tilde{a}_{i j} \\
& D_{1} \tilde{v}=d_{i j}
\end{aligned}
$$

Since (A.25) is also valid for $\hat{a}_{i j}=0$, there exists a vector $v$ such that

$$
\begin{aligned}
& \left(A_{1}+B_{1} F_{1}-\lambda_{i}\right) v=a_{i j} \\
& D_{1} v=d_{i j}
\end{aligned}
$$

Define $\hat{v} \equiv \tilde{v}-v$ and subtract to obtain 


$$
\begin{aligned}
& \left(A_{1}+B_{l} F_{1}-\lambda_{i}\right) \hat{v}=\delta \hat{a}_{i j} /\|\hat{A}\| \\
& D_{1} \hat{v}=0
\end{aligned}
$$

for $0 \leq \delta<\delta_{0}$ and $\hat{a}_{i j}$ arbitrary. There can be a solution $\hat{v}$ for these equations for each possible value of $\delta$ and árbitrary $\hat{a}_{i j}$ only if

$$
x_{1} \subset\left(A_{1}+B_{1} F_{1}-\lambda_{i}\right) \operatorname{Ker} D_{1}
$$

which is obviously impossible. Thus the original assumption was false.

$$
\text { Q.E.D. }
$$

\section{Proof of Theorem 4.2}

(nec) Given $\left(A_{1}, B_{1}, C_{1}\right)$ stabilizable and detectable, it is straightforward to show that there exists a $\delta_{0}>0$ such that $\left(\tilde{A}_{1}, \tilde{B}_{1}, C_{1}\right)$ is stabilizable and detectable for $0 \leq \delta<\delta_{0}$ and arbitrary $\left(\hat{A}_{1}, \hat{B}_{1}\right)$. Thus there exists an $F_{l}$ such that

$$
\sigma\left(\tilde{A}_{1}+\tilde{B}_{1} F_{1}\right) \subset C^{-} \quad 0 \leq \delta<\delta_{0}
$$

Now assume that (4.3) is true. Then for any $i \in \bar{r}, j \in \bar{q}_{i}$, the first equation of (4.3) yields

$$
\operatorname{rank}\left[\begin{array}{c}
\left(\tilde{A}_{1}+\tilde{B}_{1} F_{1}-\lambda_{i} 1\right) \\
D_{1}
\end{array}\right]=n_{1}=\operatorname{rank}\left[\begin{array}{cc}
\left(\tilde{A}_{1}+\tilde{B}_{1} F_{1}-\lambda_{i} 1\right) & \tilde{B}_{1} \\
D_{1} & 0
\end{array}\right]
$$

for $0 \leq \delta<\delta_{0}$. This implies that there exists a matrix $\tilde{v}^{l}$ such that

$$
\begin{aligned}
& \left(\tilde{A}_{1}+\tilde{B}_{1} F_{1}-\lambda_{i} 1\right) \tilde{V}^{1}=\tilde{B}_{1} \\
& D_{1} \tilde{V}^{1}=0
\end{aligned}
$$

By (A.26) $\left(\tilde{A}_{1}+\tilde{B}_{1} F_{1}-\lambda_{i} 1\right)$ is invertible for $0 \leq \delta<\delta_{0}$ and so $\tilde{V}^{1}$ can be written as

$$
\tilde{v}^{1}=v_{0}^{1}+\delta v_{1}^{1}+\delta^{2} v_{2}^{1}+\ldots
$$


where the $V_{\ell}^{l}$ are constant matrices. Equation (A.28) becomes

$$
\begin{aligned}
& A_{\lambda_{i}}\left(1-\delta L_{11}\right)\left(V_{0}^{l}+\delta V_{1}^{1}+\delta^{2} V_{2}^{l}+\ldots\right)=B_{1}+\delta \hat{B}_{1} /\|B\| \\
& D_{1}\left(V_{0}^{1}+\delta V_{1}^{1}+\delta^{2} V_{2}^{1}+\ldots\right)=0
\end{aligned}
$$

for $0 \leq \delta<\delta \delta_{0}$ where $A_{\lambda_{i}}$ and $L_{\|}$were defined in Theorem 4.2. Writing the equations as polynomials in $\delta$ gives us

$$
\begin{array}{ll}
v_{0}^{l}=A_{\lambda_{i}}^{-1} B_{l} & D_{1} V_{0}^{l}=0 \\
v_{l}^{l}=L_{11} V_{0}^{l}+A_{\lambda_{i}}^{-1} \hat{B}_{l} /\|\hat{B}\| & D_{1} V_{l}^{l}=0 \\
v_{l}^{l}=L_{11} V_{l-1}^{l} & D_{1} V_{l}^{l}=0 \quad l=2,3, \ldots
\end{array}
$$

From the second equation of (4.3)

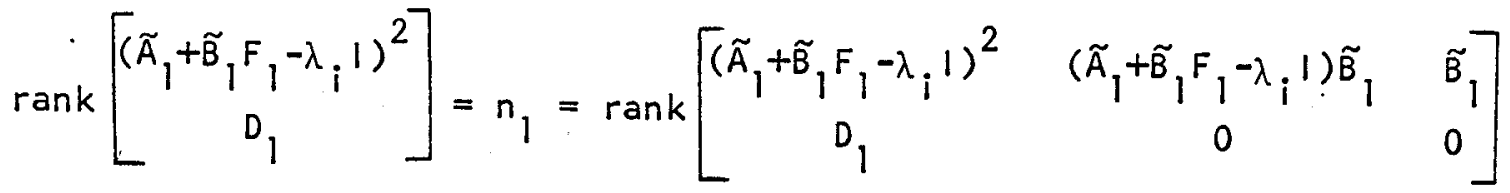

$$
\begin{aligned}
& =\operatorname{rank}\left[\begin{array}{cc}
\left(\tilde{A}_{1}+\tilde{B}_{1} F_{1}-\lambda_{i} l\right)^{2} & \tilde{B}_{1} \\
D_{1} & 0
\end{array}\right]
\end{aligned}
$$

using (A.27). Equation (A.30) implies the existence of a matrix $\tilde{V}^{2}$ such that

$$
\begin{aligned}
& \left(\tilde{A}_{1}+\tilde{B}_{1} F_{1}-\lambda_{i} 1\right)^{2} \tilde{V}^{2}=\tilde{B}_{1} \\
& D_{1} \tilde{V}^{2}=0
\end{aligned}
$$

which can be written as

$$
\begin{aligned}
& A_{\lambda_{i}}^{2}\left(1-\delta L_{21}-\delta^{2} L_{22}\right)\left(v_{0}^{2}+\delta v_{1}^{2}+\delta^{2} v_{2}^{2}+\ldots\right)=B_{1}+\delta \hat{B}_{1} /\|\hat{B}\| \\
& D_{1}\left(v_{0}^{2}+\delta v_{1}^{2}+\delta^{2} v_{2}^{2}+\ldots\right)=0
\end{aligned}
$$

for $0 \leq \delta<\delta_{0}$. Writing this as polynomials in $\delta$ clearly gives 


$$
\begin{array}{ll}
v_{0}^{2}=A_{\lambda_{i}}^{-2} B_{1} & D_{1} v_{0}^{2}=0 \\
v_{1}^{2}=L_{21} v_{0}^{2}+A_{\lambda_{i}}^{-2} B_{1} /\|B\| & D_{1} v_{l}^{2}=0 \\
v_{l}^{2}=L_{21} v_{l-1}^{2}+L_{22} v_{l-2}^{2} & D_{1} v_{l}^{2}=0 \quad l=2,3, \ldots
\end{array}
$$

This technique can be followed for the rest of equation (4.3) to establish the remainder of the equations of (4.4) that are associated with the $v_{l}^{t}$.

Observe that the first equation of (4.3) also yields

$$
\operatorname{rank}\left[\begin{array}{c}
\left(\tilde{A}_{1}+\tilde{B}_{1} F_{1}-\lambda_{i} 1\right) \\
D_{1}
\end{array}\right]=n_{1}=\operatorname{rank}\left[\begin{array}{ccc}
\left(\tilde{A}_{1}+\tilde{B}_{1} F_{1}-\lambda_{i} l\right) & \tilde{B}_{1} & \tilde{a}_{i j} \\
D_{1} & 0 & d_{i j}
\end{array}\right]
$$

and upon using $(A .27)$, this becomes

$$
\operatorname{rank}\left[\begin{array}{c}
\left(\tilde{A}_{1}+\tilde{B}_{1} F_{1}-\lambda_{i} 1\right) \\
D_{1}
\end{array}\right]=\operatorname{rank}\left[\begin{array}{cc}
\left(\tilde{A}_{1}+\tilde{B}_{1} F_{1}-\lambda_{i} 1\right) & \tilde{a}_{i j} \\
D_{1} & d_{i j}
\end{array}\right]
$$

Then in a manner analogous to that used to derive (A.29), the equations for $W_{l}^{l}$ of (4.4) can be obtained. Similarly from (4.3)

$$
\operatorname{rank}\left[\begin{array}{c}
\left(\tilde{A}_{1}+\tilde{B}_{1} F_{1}-\lambda_{i} 1\right)^{2} \\
D_{1}
\end{array}\right]=\operatorname{rank}\left[\begin{array}{cc}
\left(\tilde{A}_{1}+\tilde{B}_{1} F_{1}-\lambda_{i} 1\right)^{2} & \tilde{a}_{i j} \\
D_{1} & 0
\end{array}\right]
$$

and the equations for the $w_{l}^{2}$ can be derived. This process is continued in the same manner to obtain the remainder of (4.3)

(suff) The sufficiency of (4.4) is established by essentially applying, in reverse, the technique of the necessity proof. 'Assume (4.4) is true, then for $t=1$ we have

$$
D_{1} v_{l}^{l}=0 \quad \ell=1,2, \ldots, n_{l}
$$


39

where

$$
\begin{array}{ll}
v_{0}^{l}=A_{\lambda_{i}}^{-1} B_{1} & v_{1}^{l}=L_{\| 1} V_{0}^{1}+A_{\lambda_{i}}^{-1} \hat{B}_{1} /\|B\| \\
v_{l}^{\prime}=L_{11} V_{l-1}^{l} & l \geq 2
\end{array}
$$

Observe that $v_{l}^{\prime}$ may be written as

$$
v_{l}^{l}=\left(L_{11}\right)^{l-1} v_{1}^{l} \quad \text { for } l \geq 1
$$

and by (A.32)

$$
D_{1}\left(L_{11}\right)^{l} v_{1}^{\prime}=0 \quad l=0,1, \ldots, n_{1}-1
$$

Since $L_{11}$ is an $n_{1} \times n_{1}$ matrix, the Cayley-Hamilton theorem can be used. to extend the index of (A.32). The solvability of RSFP yields $D_{1} V_{0}^{1}=0$ and

so

$$
D_{1} v_{l}^{l}=0 \quad l=0,1,2, \ldots
$$

and the reverse of the arguments leading to (A.29) can be applied to obtain

$$
n_{1}=\operatorname{rank}\left[\begin{array}{cc}
\left(\tilde{A}_{1}+\widetilde{B}_{1} F_{1}-\lambda_{i}{ }^{1}\right) & \tilde{B}_{1} \\
D_{1} & 0
\end{array}\right]=\operatorname{rank}\left[\begin{array}{cc}
\left(\tilde{A}_{1}-\lambda_{i} 1\right) & \tilde{B}_{1} \\
D_{1} & 0
\end{array}\right] \quad 0 \leq \delta<\delta
$$

For $t=2$ we have from $(4.3)$

$$
D_{1} v_{l}^{2}=0
$$

$$
l=1,2, \ldots, 2 n_{1}
$$

where

$$
\begin{array}{ll}
v_{0}^{2}=A_{\lambda_{i}}^{-2} B_{1} & v_{1}^{2}=L_{21} v_{0}^{2}+A_{\lambda_{i}}^{-2} \hat{B}_{1} / \| \hat{B}_{\|} \\
v_{l}^{2}=L_{21} v_{l-1}^{2}+L_{22} v_{l-2}^{2} & l \geq 2
\end{array}
$$

Observe that for $l \geq 1$ 


$$
\left[\begin{array}{c}
v_{l}^{2} \\
v_{l}^{1}
\end{array}\right]=\left[\begin{array}{ll}
L_{21} & L_{22} \\
1 & 0
\end{array}\right]^{\ell-1}\left[\begin{array}{l}
v_{1}^{2} \\
v_{0}^{2}
\end{array}\right]
$$

and

$$
\left[\begin{array}{ll}
0, & 0
\end{array}\right]\left[\begin{array}{ll}
L_{21} & L_{22} \\
1 & 0
\end{array}\right]^{l-1}\left[\begin{array}{l}
v_{1}^{2} \\
v_{0}^{2}
\end{array}\right]=0 \quad . \quad l=0,1, \ldots, 2 n_{1}-1
$$

Since $\left[\begin{array}{ll}L_{21} & L_{22} \\ l & 0\end{array}\right]$ is a $2 n_{1} \times 2 n_{1}$ matrix, the Cayley-Hamilton theorem can be used to extend the index of (A.34) and, combining this with the solvability of RSFP, we have

$$
D_{1} v_{l}^{2}=0 \quad l=0,1,2, \ldots
$$

Applying the reverse of the ideas leading to (A.31) gives us

$$
n_{1}=\operatorname{rank}\left[\begin{array}{c}
\left(\tilde{A}_{1}+\tilde{B}_{1} F_{1}-\lambda_{i} 1\right)^{2} \\
D_{1}
\end{array}\right]=\operatorname{rank}\left[\begin{array}{cc}
\left(\tilde{A}_{1}+\tilde{B}_{1} F_{1}-\lambda_{i} \mid\right)^{2} & \tilde{B}_{1} \\
D_{1} & 0
\end{array}\right] \quad 0 \leq \delta<\delta_{0}
$$

and combining this wi th (A.33)

$$
n_{1}=\operatorname{rank}\left[\begin{array}{ccc}
\left(\widetilde{A}_{1}+\widetilde{B}_{1} F_{1}-\lambda_{i} \mid\right)^{2} & \left(\widetilde{A}_{1}+\tilde{B}_{1} F_{1}-\lambda_{i} 1\right) \tilde{B}_{1} & \widetilde{B}_{1} \\
D_{1} & 0 & 0
\end{array}\right] \quad 0 \leq \delta<\delta_{0}
$$

which implies

$$
n_{1}=\operatorname{rank}\left[\begin{array}{ccc}
\left(\tilde{A}_{1}-\lambda_{i} 1\right)^{2} & \left(\tilde{A}_{1}-\lambda_{i} 1\right) \tilde{B}_{1} & \tilde{B}_{1} \\
D_{1} & 0 & 0
\end{array}\right] \quad 0 \leq \delta<\delta_{0}
$$

This same technique can be applied to the remainder of (4.4) which establishes (4.3) and proves the theorem. 